\title{
Theory and practice of orbital angular momentum and beyond
}

\author{
Abderrahmen Trichili, Mitchell A. Cox, Benjamin Perez-Garcia, Boon S. Ooi, and \\ Mohamed-Slim Alouini
}

\begin{abstract}
Nearly three decades since its discovery, orbital angular momentum (OAM) has proven to be highly versatile for a wide range of applications. It is an indispensable tool in quantum optics, has made a significant impact in optical tweezing, enabled higher contrast and more detailed imaging, and offers a convenient way to harness the space degree of freedom in telecommunications. In this paper, we present a review of a wide range of applications of OAM as well as describing the creation and detection of OAM modes, with a focus on the use of OAM in communications. In addition, we detail various similar higher-order optical modes, such as vector vortex modes, and provide an introduction to the use of OAM in quantum optics, pitched for readers new to the field.
\end{abstract}

\section{INTRODUCTION}

In 1992, Allen et al. discovered that light can carry orbital angular momentum (OAM) [1]. This is a fundamental property of light, with each photon having $\ell \hbar$ of orbital angular momentum, where $\hbar$ is the reduced Planck constant. OAM is different from spin angular momentum (SAM), associated with the polarization of light. SAM can only take two states: right-handed and left-handed. On the other hand, OAM is defined by a so-called topological charge (or azimuthal mode index), $\ell$, which is an unbounded integer and represents the number of $2 \pi$ phase rotations in one wavelength. In a classical context, OAM manifests as a helical wavefront and is characterized by an azimuthally varying phase term $\exp (i \ell \phi)$. Here, $\phi$ is the azimuthal angle.

The wavefront of an OAM beam of light is twisted like a corkscrew, or perhaps a Fusilli pasta. Since the phase at the center is undefined, there can be no light in the center, and so the beam possesses a doughnut-like transverse intensity profile, as can be seen in Fig. 1.

The orbital angular momentum of light has proven to be highly versatile and has unlocked many new avenues of research. It has found use in astronomy [2], particle manipulation and tweezing [3], imaging [4], remote sensing [5], quantum optics [6], and communication technology [7], [8], for example.

Here we particularly focus on the contribution of OAM in communication and quantum information. We begin by describing some of the more common higher-order mode sets, some of which contain OAM in Sec. III Following this, in Sec. III we delve into more detail on the various methods and techniques used for the generation and detection of OAM modes in particular. We identify and briefly describe the main contributions of OAM across multiple communication forms, namely free space optics (FSO), optical fiber,

A. Trichili, B. S. Ooi, and M.-S. Alouini are with the Computer, Electrical and Mathematical Sciences \& Engineering in King Abdullah University of Science and Technology, Thuwal, Makkah Province, Saudi Arabia. (email: abderrahmen.trichili,boon.ooi,slim.alouini@kaust.edu.sa)

M. A. Cox is with the School of Electrical and Information Engineering, University of the Witwatersrand, Johannesburg, South Africa. (email: Mitchell.Cox@wits.ac.za).

B. Perez-Garcia is with Photonics and Mathematical Optics Group, Tecnologico de Monterrey, Monterrey 64849, Mexico (email: b.pegar@tec.mx). 
(a)

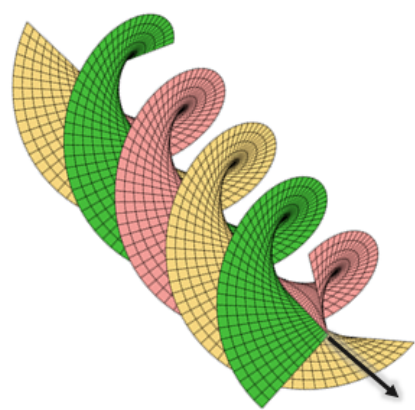

(c)

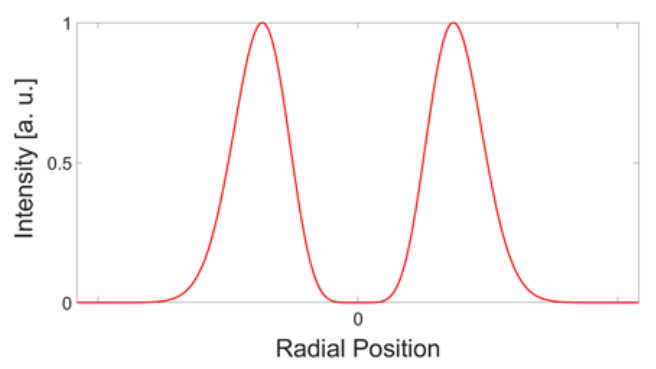

(b)

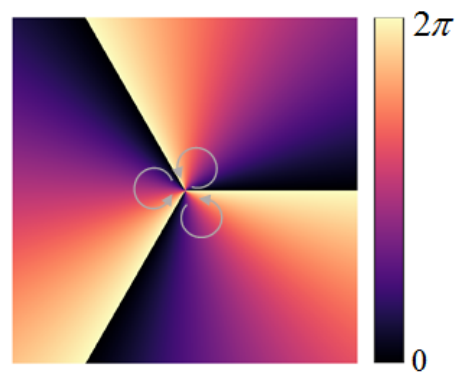

(d)

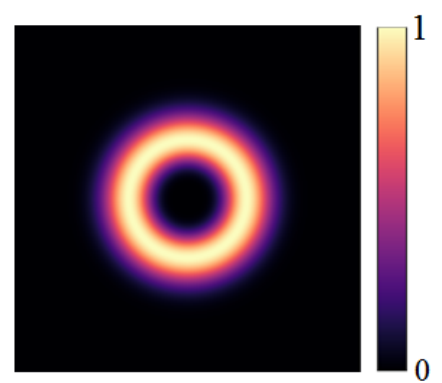

Fig. 1. A schematic illustration (a) helical phasefront, (b) transverse phase profile, (c,d) intensity, and transverse intensity profile of an OAM with $\ell=3$.

underwater optical wireless communication (UWOC), and radio frequency (RF) systems in Sec. IV. In $\mathrm{Sec}$. IV, we also discuss the major practical implementation challenges of OAM communication links and the different mitigation strategies. Vector OAM modes, or in other words, OAM beams with a non-uniform polarization, are a very interesting family of modes with novel applications and properties. We discuss some of these applications and important lab techniques and theory applicable to these so-called vector vortex modes in Sec. V]. Finally, OAM has, in some sense, revolutionized the tools available to us in the quantum world. We describe these tools and applications in Sec. VI.

\section{Higher-Order Spatial Modes of Light}

Electromagnetic fields in free space can be described by the Helmholtz equation where the complex amplitude is that of the electric vector field $(\mathbf{E})$ :

$$
\left[\nabla^{2}+n^{2} k^{2}\right] \mathbf{E}=0
$$

where $k$ is the wave number in a vacuum, and $n$ is the refractive index ( $n=1$ in a vacuum). The wavenumber is defined as:

$$
k=\frac{2 \pi}{\lambda},
$$

where $\lambda$ is the wavelength. Solutions to the Helmholtz equation (1) are often referred to as modes.

It is worth noting that any complex-valued function can be written in the form:

$$
g(\mathbf{s}, z)=A(\mathbf{s}, z) e^{i \theta(\mathbf{s}, z)},
$$


where $\mathbf{s}$ refers to the transverse coordinates, which can be Cartesian, $s \equiv(x, y)$ or cylindrical, $s \equiv(r, \phi)$. $A(\mathbf{s}, z)$ is called the amplitude function or magnitude of the complex function. $\theta(\mathbf{s}, z)$ is called the phase function, which is the argument of the complex function.

If we assume that the propagation of an optical beam is mainly in the $z$ direction, known as the paraxial approximation, then it is convenient to write the scalar field vector as:

$$
U(\mathbf{s}, z)=A(\mathbf{s}, z) e^{-i k z},
$$

where the transverse profile of the beam is a complex scalar wave amplitude, $A$. For convenience, we can split the Laplacian operator into a transverse $(T)$ and $z$ component, resulting in:

$$
\nabla^{2}=\nabla_{T}^{2}+\delta_{z}^{2}
$$

If Equation (4) is substituted into Equation (1) and the split Laplacian is used, the resulting Helmholtz equation becomes:

$$
\nabla_{T}^{2} A(\mathbf{s}, z)+\frac{\delta^{2} A(\mathbf{s}, z)}{\delta z^{2}}-2 i k \frac{\delta A(\mathbf{s}, z)}{\delta z}=0
$$

Since the propagation is mainly in the $z$ direction, this results in a very slow variation in the $z$ direction, we can assume that $\delta_{z}^{2} A(\mathbf{s}, z) \approx 0$ and so under the paraxial approximation the equation becomes:

$$
\nabla_{T}^{2} A(\mathbf{s}, z)-2 i k \frac{\delta A(\mathbf{s}, z)}{\delta z}=0
$$

\section{A. Laguerre Gaussian Modes}

Orbital angular momentum modes are derived from the Laguerre Gaussian (LG) mode family, which represents a solution to the Helmholtz equation under the paraxial wave approximation. The electric field of an LG mode with a topological charge $\ell$ and radial index $p$ in a cylindrical system coordinates $(r, \phi, z)$, is given as follows:

$$
\begin{aligned}
E_{(p, \ell)}^{L G}(r, \phi, z)= & \frac{1}{\omega(z)} \sqrt{\frac{2 p !}{\pi(|\ell|+p) !}} \exp [i(2 p+|\ell|+1) \Phi(z)]\left(\frac{\sqrt{2} r}{\omega(z)}\right)^{|\ell|} L_{p}^{|\ell|}\left(\frac{2 r^{2}}{\omega(z)^{2}}\right) \\
& \times \exp \left(-\frac{i k r^{2}}{2 R(z)}\right) \exp \left(-\frac{r^{2}}{\omega(z)^{2}}\right) \exp (i \ell \phi),
\end{aligned}
$$

with

$$
\begin{gathered}
\omega(z)=\omega_{0} \sqrt{1+z^{2} / z_{R}^{2}}, \\
R(z)=z\left[1+\left(z_{R} / z\right)^{2}\right], \\
z_{R}=\pi \omega_{0}^{2} / \lambda, \\
\Phi(z)=\arctan \left(z / z_{R}\right),
\end{gathered}
$$

Here, $\omega(z)$ is the Gaussian beam width, and $\omega_{0}$ is the beam width at the origin $(z=0) . R(z)$ denotes the beam curvature and $z_{R}$ is the Rayleigh range of the beam, which is the distance along the propagation direction from $z=0$ where beam radius is increased by a factor of $\sqrt{2}$. $\Phi(z)$ is the Gouy phase. $L_{p}^{|\ell|}(\cdot)$ are the generalized Laguerre Polynomials. $k=2 \pi / \lambda$ is the wavenumber, and $\lambda$ is the operating wavelength. The particular case of $\ell=p=0$ corresponds to the Gaussian beam. The $M^{2}=2 p+|\ell|+1$ is the beam propagation factor of an LG mode, which describes how the beam propagates in free space. 

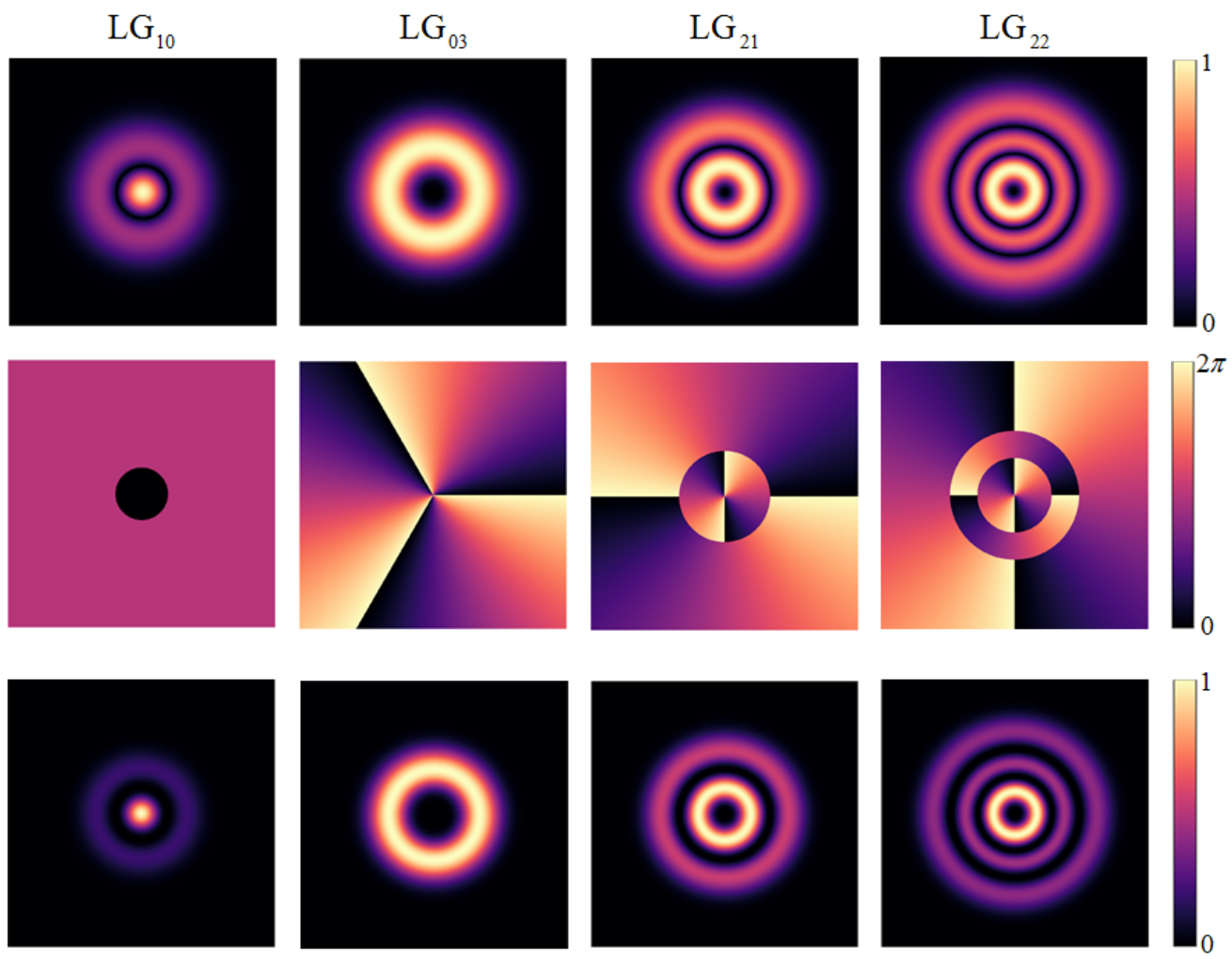

Fig. 2. A set of (top) amplitude, (middle) phase and (bottom) intensity profiles of LG modes.

The transverse intensity profiles of LG beams are given in Fig. 2. LG modes with $p=0$ are commonly known as OAM-only beams. At any $z$ plane, LG beams, with the same waist are orthogonal with respect to $\ell$ and $p$ :

$$
\begin{aligned}
\left\langle E_{(p, \ell)}^{L G}, E_{\left(p^{\prime}, \ell^{\prime}\right)}^{L G}\right\rangle= & \int_{0}^{\infty} \int_{0}^{2 \pi} E_{(p, \ell)}^{L G} E_{\left(p^{\prime}, \ell^{\prime}\right)}^{L G} r d r d \phi \\
& =\delta_{\ell \ell^{\prime}} \delta_{p p^{\prime}}=\left\{\begin{array}{ll}
0 & \ell \neq \ell^{\prime} \text { or } p \neq p^{\prime} \\
1 & \ell=\ell^{\prime} \text { and } p=p^{\prime}
\end{array},\right.
\end{aligned}
$$

where $\langle\cdot\rangle$ is the inner product operator and ${ }^{*}$ denotes the complex conjugate. Any beam can be decomposed in the LG mode basis as follows:

$$
U=\sum_{n, m} c_{n m} E_{n, m}^{L G}
$$

where $c_{n m}$ are the mode content coefficients (these are complex numbers to represent relative amplitude and phase) and can be determined through inner product measurements:

$$
c_{n m}=\left\langle U, E_{n, m}^{L G}\right\rangle \text {. }
$$

\section{B. Hermite Gaussian Beams}

Hermite Gaussian beams are solutions of the paraxial wave equation. Each $\mathrm{HG}_{n_{x} n_{y}}$ mode is characterized by two indices $n_{y}$ and $n_{y}$, which indicate the number of nodes on the horizontal and the vertical axis, 
respectively. In a Cartesian coordinate system $(x, y, z)$, the electric field of a Hermite Gaussian beam can be written as [9]:

$$
\begin{aligned}
E_{\left(n_{x}, n_{y}\right)}^{H G}(x, y, z) & =\sqrt{\frac{2}{\pi n_{x} ! n_{y} !}} 2^{-\frac{n_{x}+n_{y}}{2}} \exp \left[-i \frac{k\left(x^{2}+y^{2}\right)}{2 R(z)}\right] \exp \left[i\left(n_{x}+n_{y}+1\right) \Phi(z)\right] \\
& \times \exp \left(-\frac{x^{2}+y^{2}}{\omega(z)^{2}}\right) H_{n_{x}}\left(\frac{\sqrt{2} x}{\omega(z)}\right) H_{n_{y}}\left(\frac{\sqrt{2} y}{\omega(z)}\right),
\end{aligned}
$$

where $H_{n_{x}}(\cdot)$ and $H_{n_{y}}(\cdot)$ are Hermite polynomials of order $n_{x}$ and $n_{y}$, respectively. The beam quality factor of a HG beam is $M^{2}=n_{x}+n_{y}+1$ and the particular case of $n_{x}=n_{y}=0$ corresponds to the Gaussian beam. Figure 3 contains the amplitude, phase and intensity of a set of HG modes.
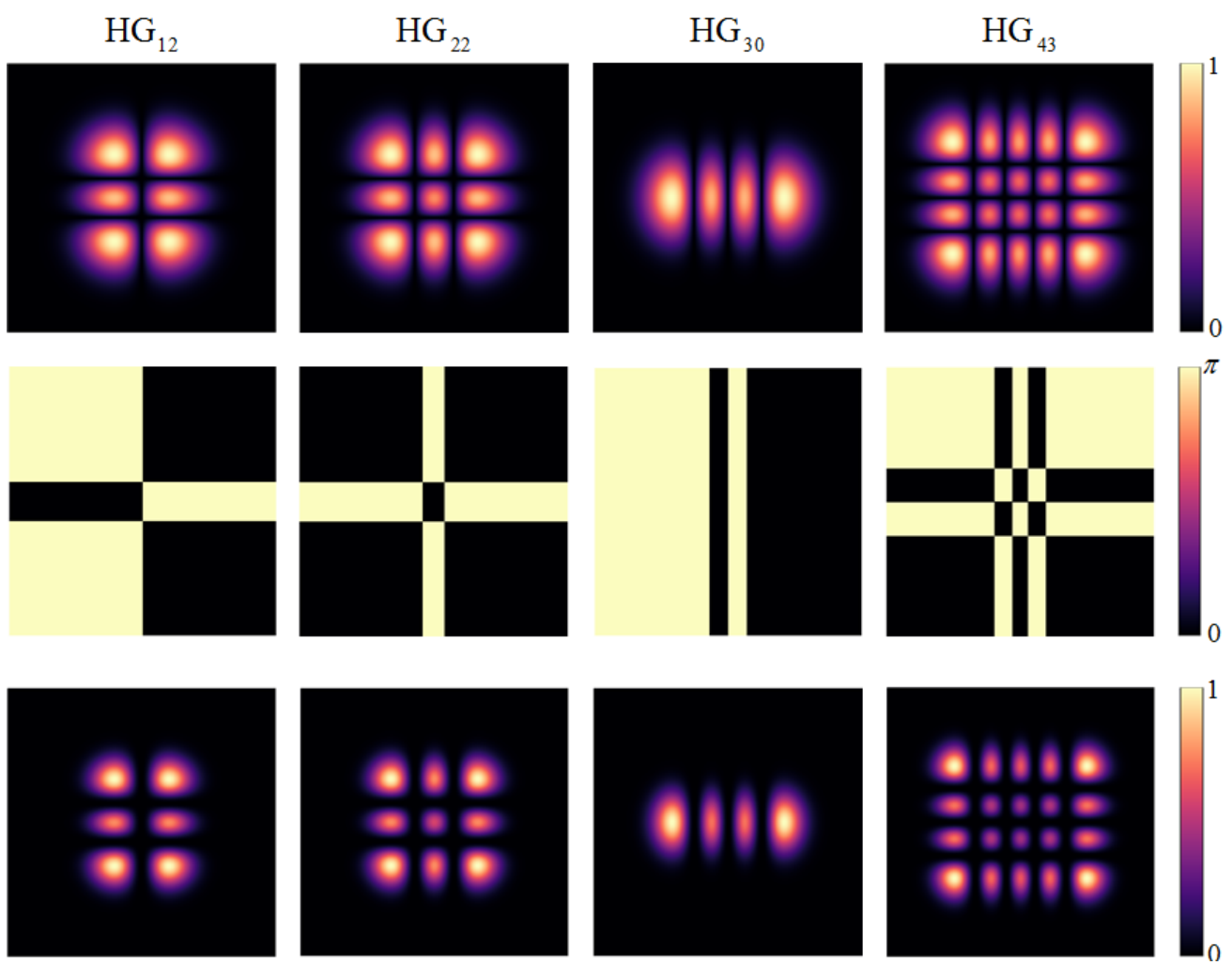

Fig. 3. A set of (top) amplitude, (middle) phase and (bottom) intensity profiles of HG modes.

Since HG and LG both form orthonormal and complete mode basis, any mode from the HG basis can be expressed as a function of LG modes, and vice versa. Expressing LG as a function of HG modes can be as follows [10]:

$$
E_{n m}^{L G}=\sum_{k=0}^{N} i^{k} b(n, m, k) E_{N-k, k}^{H G}
$$

with

$$
b(n, m, k)=\left.\left(\frac{(N-k) ! k !}{2^{N} n ! m !}\right)^{1 / 2} \frac{1}{k !} \frac{d^{k}}{d t^{k}}\left[(1-t)^{n}(1+t)^{m}\right]\right|_{t=0},
$$

where $N=n+m=2 p+|\ell|$. The indices $(n, m)$ used here for the LG mode are not the same as $\ell$ and $p$ initially defined in section II-A, which are $p=\min (n, m)$ and $\ell=n-m$. Figure 4 shows the decomposition of LG modes in terms of HG modes. Transforming HG modes to LG modes (and from 


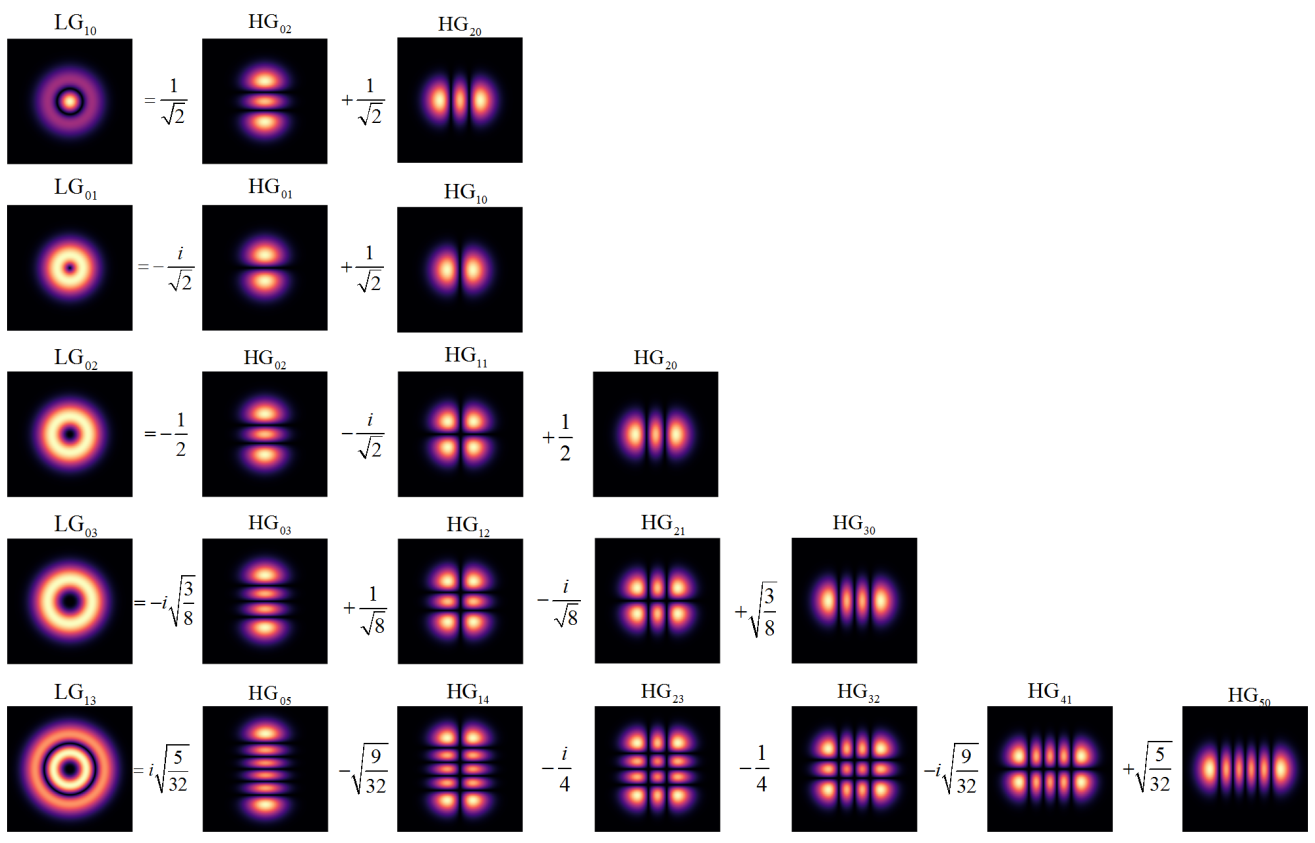

Fig. 4. Decomposition of LG beams with $(p, \ell)$ indices as a function of HG modes with $(n, m)$ indices, determined using Eq. 17.

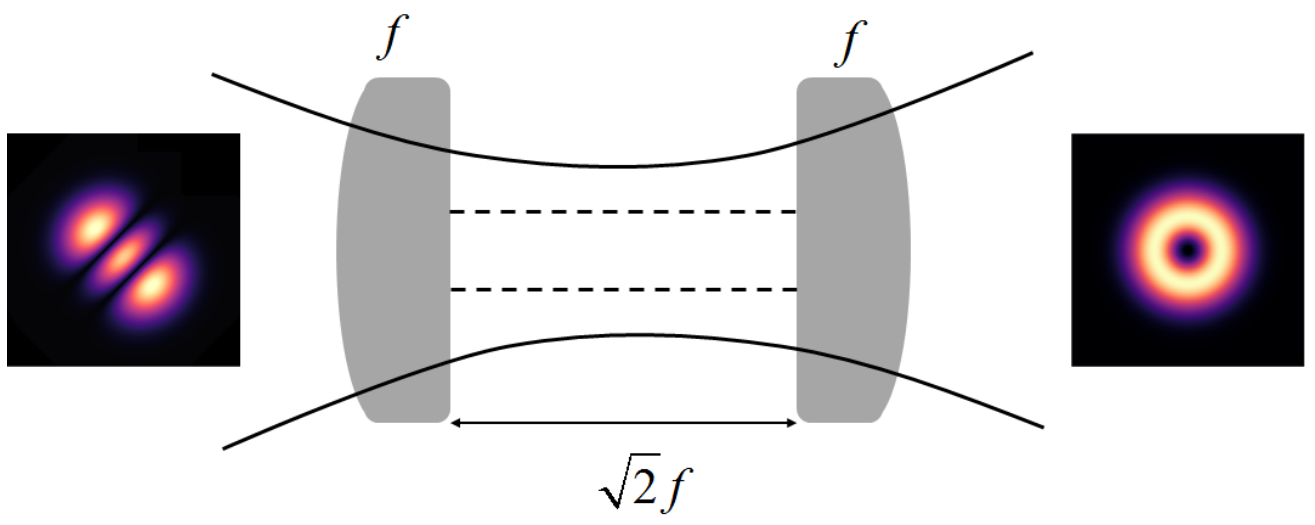

Fig. 5. HG to LG mode conversion using cylindrical lenses. $f$ is the focal length of each of the cylindrical lenses.

LG to HG) can be performed experimentally via cylindrical lenses, as demonstrated in [10]. An example of HG beam conversion to an LG beam using 2 cylindrical lenses is shown in Fig. 5 .

\section{Ince Gaussian Beams}

Ince Gaussian (IG) modes are another family of modes that are exact solutions of the paraxial wave approximation in elliptical coordinates [11]. Each mode is characterized by three parameters $p, m$, and $\epsilon$. The mode indices $(p, m)$ are positive integers. $\epsilon$ defines the beam ellipticity. The electric field of an even IG beam is given as follows:

$$
E_{(p, m, \epsilon)}^{I G}(u, v, z)=A_{I G} C_{p}^{m}(i u, \epsilon) C_{p}^{m}(v, \epsilon) \exp \left(-\frac{r^{2}}{\omega(z)^{2}}\right) \exp \left(i \frac{k r^{2}}{2 R(z)}-i k z\right) \exp (-i(p+1) \Phi(z)),
$$



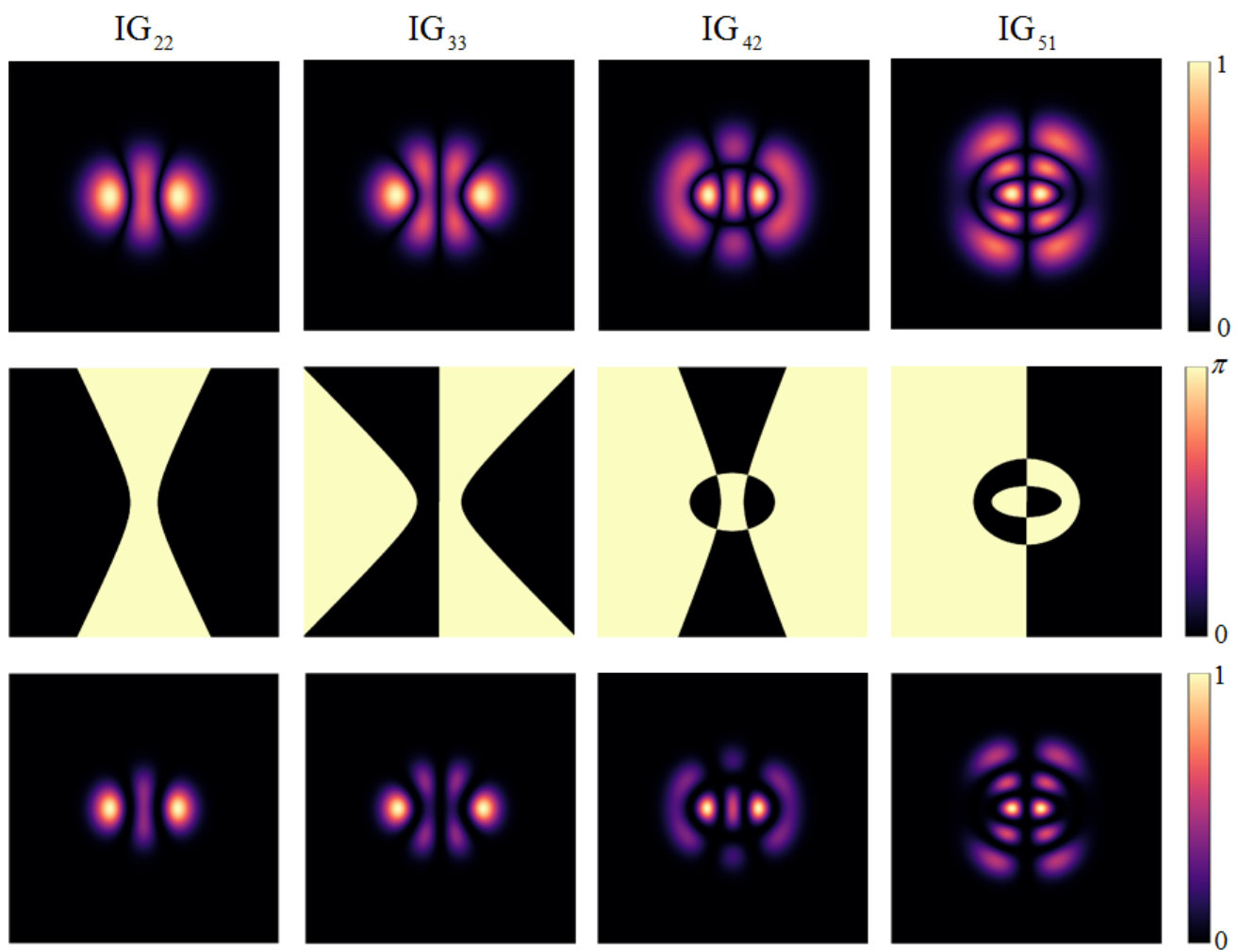

Fig. 6. A set of (top) amplitude, (middle) phase, and (bottom) intensity transverse profiles of IG beams with ellipticity parameter $\epsilon=2$.

where $A_{I G}$ is a normalization coefficient, and $C_{p}^{m}(., \epsilon)$ are the even Ince polynomials. The odd IG beams can be obtained by replacing $C_{p}^{m}(., \epsilon)$ by $S_{p}^{m}(., \epsilon)$, the odd Ince polynomials. $u$ and $v$ are the radial and angular elliptical coordinates, respectively, defined by:

$$
\begin{aligned}
& x=\sqrt{\epsilon / 2} \omega_{0} \cosh (u) \cos (v) \\
& y=\sqrt{\epsilon / 2} \omega_{0} \sinh (u) \sin (v)
\end{aligned}
$$

When the elliptical coordinates tend to cylindrical coordinates, i.e., $\epsilon \rightarrow 0$, IG transit to LG beams. IG and LG mode indices are related in this case as $|\ell|=m$ and $p=(p-m) / 2$. When the elliptical coordinates tend to Cartesian coordinates, i.e., $\epsilon \rightarrow \infty$, IG modes transit to HG modes. IG and HG mode indices are related in this case as: $n_{x}=m$ and $n_{y}=p-m$. The beam quality factor of an IG beam is $M^{2}=p+1$. The amplitude, phase, and intensity of a set of IG beams are depicted in Fig. 6.

\section{Bessel Beams}

Bessel beams form a set of solutions of the wave equation that are non-diffracting [12]. In cylindrical coordinates $(r, \phi, z)$, the electric field of a Bessel beam is expressed as:

$$
E_{\ell, k_{r}}^{B B}(r, \phi, z)=\sqrt{2 / \ell} J_{\ell}\left(\frac{z_{R} k_{r} r}{z_{R}-i z}\right) \exp \left(i \ell \phi-i k_{z} z\right),
$$

where $J_{\ell}($.$) is the Bessel function of the first kind of order \ell . k_{r}$ and $k_{z}$ are the radial and longitudinal wavenumbers, respectively. Each Bessel beam is characterized by an $\ell$ index, indicating the OAM modal content, and $k_{r}$, which determines the spacing between the intensity rings of a beam. Figure 7 shows the 


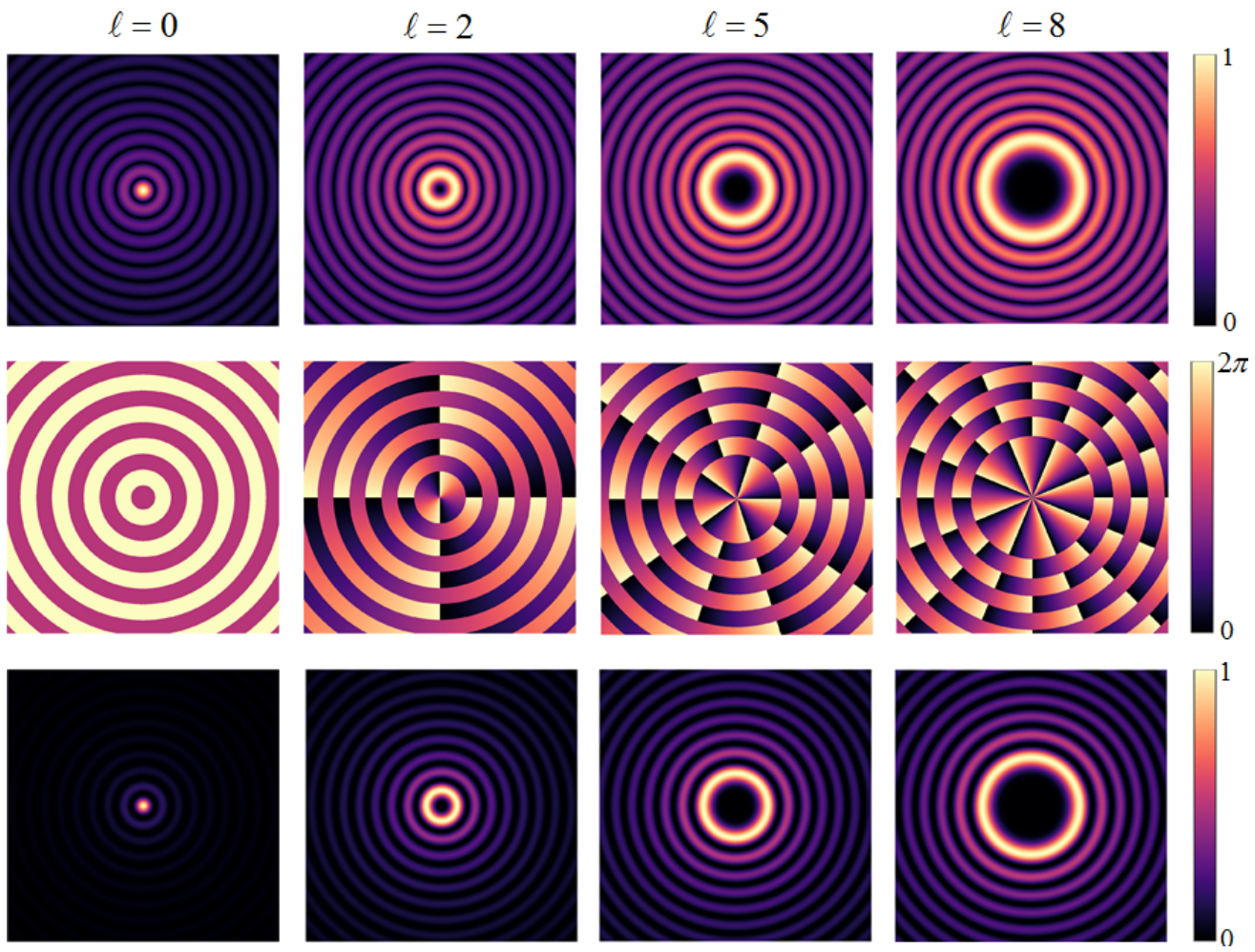

Fig. 7. A set of (top) amplitude, (middle) phase, and (bottom) intensity transverse profiles of Bessel beams.

amplitude, phase, and intensity of a set of Bessel beams. Unlike Gaussian beams, a Bessel beam carry an infinite amount of power. In practice, it is not possible to generate beams with infinite power, therefore, Bessel beams are approximated with Bessel Gaussian (BG) beams, whose electric fields can be expressed as follows:

$$
E_{\ell, k_{r}}^{B G}(r, \phi, z)=\sqrt{2 / \ell} J_{\ell}\left(\frac{z_{R} k_{r} r}{z_{R}-i z}\right) \exp \left(i \ell \phi-i k_{z} z\right) \exp \left(\frac{i k_{r}^{2} z \omega_{0}^{2}-2 k r^{2}}{4\left(z_{R}-i z\right)}\right),
$$

BG beams are able to reconstruct themselves after encountering an obstruction, along the propagation path. However, when generate in laboratory, they have a finite propagation length, given by:

$$
z_{\max }=\frac{2 \pi \omega_{0}}{\lambda k_{r}}
$$

Note that in addition to the LG, HG, IG and BG mode bases, other bases are solutions to the wave equation, including the Mathieu and Airy mode families, which can carry OAM but will not be covered here. In the following, we focus on OAMs derived from the LG mode family.

\section{OAM GENERATION AND DETECTION}

As we discussed in II-B, mode converters based on cylindrical lenses can take as input HG laser modes to produce LG modes carrying OAMs. OAMs can be also generated using various other techniques, including spiral phase plates (SPPs) [13], q-plates [14], computer-generated holograms (CGHs) printed on spatial light modulators (SLMs) [15]: 


\section{A. Spiral Phase Plates}

A SPP is a structure with a thickness that varies with the azimuthal angle and introduces a phase shift of $2 \pi \ell$ to an incoming Gaussian light beam, as can be seen in Fig. 8(a1). To generate an OAM with a charge $\ell$, an SPP is designed in a way that the step height, the maximum thickness difference between the lowest and highest point, is $h_{s}=\ell \lambda /\left(n-n_{0}\right)$, with $\lambda$ is the incident light wavelength, $n$ is the refractive index of the material from which the SPP is fabricated, $n_{0}$ is the medium surrounding the SPP ( $n=1$ for air), and $\phi$ is the azimuth. The total height of an SPP is $h=h_{s}+h_{0}$, with $h_{0}$ is the SPP base height. SPPs can also be created using multiple "staircases". The number of etched staircases around $360^{\circ}$ in a single SPP corresponds to the absolute value of the topological charge (see the SPPs with different orders in Figs. 8(a2-a4)). The height of a unit step of an SPP with multiple staircases is $s=\lambda \phi /\left(2 \pi\left(n-n_{0}\right)\right)$ and the maximum thickness difference between the lowest and highest point is $\Delta h=\lambda /\left(n-n_{0}\right)$. Once it is fabricated, the OAM order of an SPP is fixed, and only a single OAM beam can be generated from a Gaussian input beam.

\section{B. Q-Plates}

A q-plate is an optics that takes as an input a light beam with a circular polarization state to produce an OAM beam with an opposite circular polarization state, as depicted in Fig. 8.(b). A q-plate with order $q$ generates an OAM of $\pm m \hbar$, with $m=2 q$ (depending on the handedness of the input circular polarization, as seen Fig. 8(b)). Q-plates can be fabricated using liquid crystals, computer-generated sub-wavelength gratings, or polymer materials. A single q-plate can generate a single or multiple OAM if it is tunable [16]. Static q-plates are designed to operate for a single wavelength, and liquid-crystal ones can have a working wavelength range.

\section{Holograms}

CGHs can be used to create OAM beams with different topological charges when loaded on an SLM, which is an electronically programmable device that can be set to manipulate the phase (and the amplitude) of a light beam. An illustration of OAM generation using a hologram is shown in Fig. 8(c1). CGHs are generated using a transmittance function of $\exp (i \ell \phi)$, and can also have grating functions to separate the diffraction orders, resulting in fork holograms. Fork holograms can also be binary. The spiral, fork, and binary CGHs that can be used to generate an OAM of charge $\ell=1$ are shown in Figs. 8(c2) and (c3). The most commonly used SLMs are based on liquid crystals (LCs). An LC-based SLM contains a screen of electrically addressable pixels in which the orientation of the liquid crystals is controlled by applying a voltage. The advantage of the use of SLMs is the broad spectrum of the devices and the ability to generate single and superposition of OAM beams (or any other beam-type as described above, for that matter) dynamically using a single device [17]. There are also SLMs that are based on digital micro-mirror devices (DMDs) and are composed of arrays of highly reflective micromirrors. Each individual micromirror forms a pixel element of the DMD array and can be rotated to an "on" or "off" degree state $\left( \pm 12^{\circ}\right.$ for most DMDs). DMD-based SLMs are able to generate OAMs in a faster manner than the LCs-based ones [18], [19]. DMDs are often widely and affordably available as commercial digital light projectors can sometimes be modified into SLMs [20].

\section{Metamaterials}

Metamaterial-based techniques were also proven to be efficient in generating OAM modes [21]. An illustration of a metamaterial-based OAM generation method is shown in Fig 8 (d). The example, shown in Fig. 8(d), corresponds to a metasurface composed with an array of plasmonic gold nano-antenna (having a sub- wavelength thickness) that takes as input circularly polarized visible light to produce OAM carrying light (with opposite circular polarization state to the input beam) [21]. 
(a1)
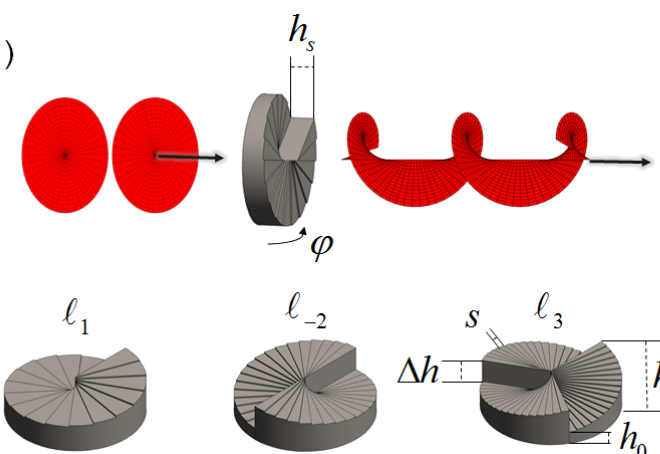

(a2)

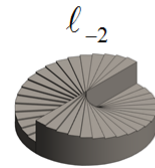

(a3)

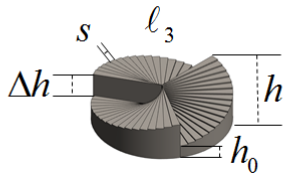

(a4)

(b)
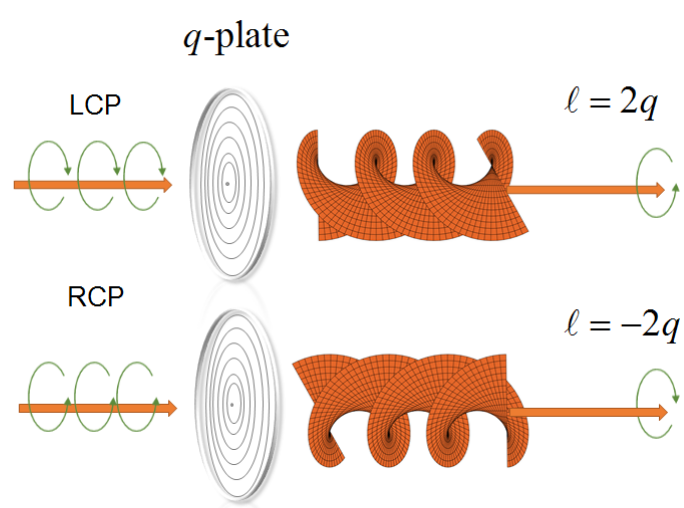

(d)
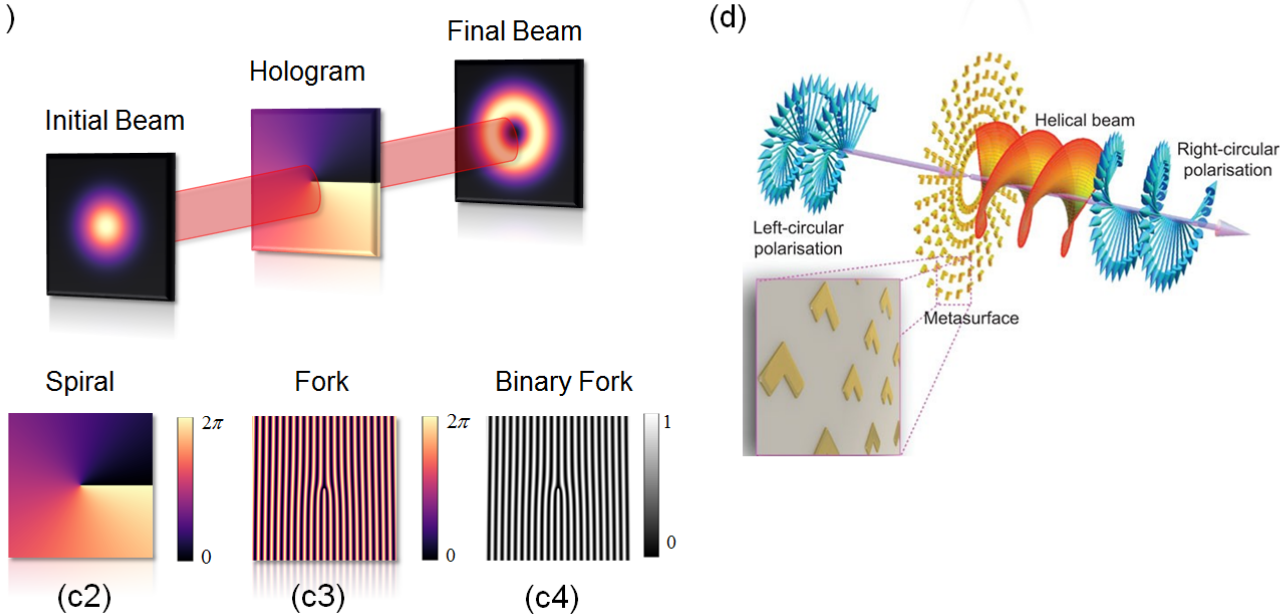

Fig. 8. An illustration of various OAM generation techniques. (a1) Creation of an OAM beam using a spiral phase plate from an incident Gaussian beam. (a2)-(a4) Examples of SPP structures with staircases. (b) Creation of an OAM using a q-plate from an incoming circularly polarized beam. (c1) OAM generation using a computer-generated hologram and Gaussian incident beam. Illustration of (c2) spiral, (c3) fork, and (c4) binary fork holograms. (d) OAM generation from an incoming circularly polarized light using a metasurface (Adapted from [21]).

\section{E. Detection Methods}

The detection of OAM beams can be accomplished using several tools. One way is to use an SPP in an inverse process to the generation, i.e., to detect an OAM of charge $\ell$, it is possible to use an SPP with an opposite charge to convert the OAM beam back to a Gaussian beam. Mode sorters based on the use of transformation optics have been proven to be efficient in measuring a large number of OAM states [22]. CGHs loaded on LCs SLMs are so far the widely commonly used method to detect OAMs with different charges and reveal the modal content of any 'unknown' beam. The technique used to characterize a beam by its modal components using CGHs is known as modal decomposition and is thoroughly explained in [23]. Interestingly, it is possible to use modal decomposition to reconstruct a beam's wavefront from its OAM and other modal components in a high-speed manner using a DMD [24].

\section{OAM IN COMMUNICATION}

OAMs form an infinite Hilbert space, which makes it attractive for communication to multiplex information in conjunction with lights other degrees of freedom: wavelength, polarization, and quadrature (phase and amplitude). Prior to the discovery of OAM, it was only possible to multiplex independent modulated data streams over different wavelengths as well as the two orthogonal polarization states. With 


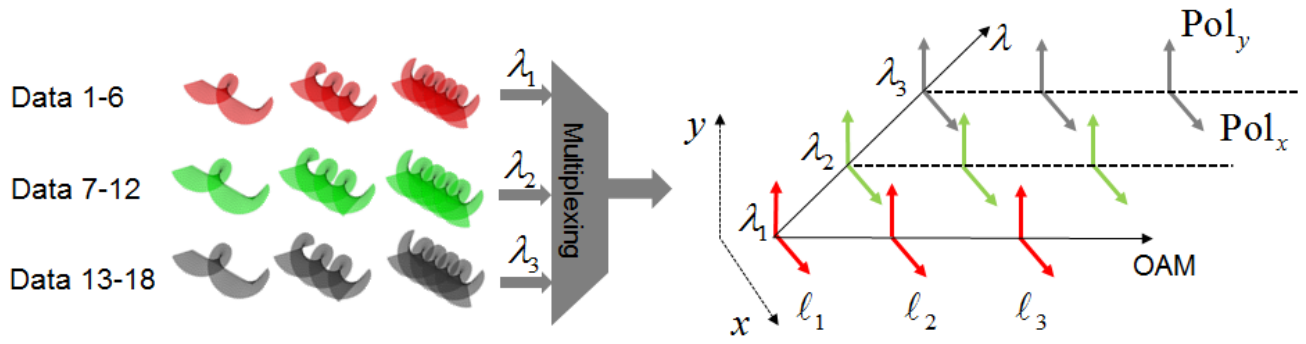

Fig. 9. An illustration of a multi-dimensional multiplexing scheme using the wavelength, polarization, and OAM degrees of freedom of light. For example, 18 data streams can be encoded using 3 OAM orders, 2 orthogonal polarization states, and 3 wavelengths.

the exponential growth of the internet, these degrees of freedom are becoming saturated (i.e., we are approaching the Shannon Limit in fiber), and so the space degree of freedom in the form of OAM is an exciting candidate to solve this issue [25]. By harnessing OAM (or any other orthogonal spatial mode for that matter), we can theoretically multiply the capacity of a communication system by the number of modes used, illustrated in Fig. 9. No system is perfect, however, and a discussion of the issues one can expect to face are in Sec. IV-B.

Instead of multiplexing, the beam's shape can also act as an information carrier, sometimes without the need for complicated or bulky detection devices or compensating for propagation effects. Only a camera is required to detect the beam shape and machine learning algorithms for beam identification. The concept can be viewed as Morse code with a theoretically infinite alphabet, instead of the 'dash' and 'dot'. Krenn et al. demonstrated in 2014 the transmission of 16 green mode shapes over a $3 \mathrm{~km}$-long turbulent channel through the city of Vienna (Austria) [26]. This work was followed by a demonstration between two Canary Islands over a total propagation distance of $143 \mathrm{~km}$ [27].

Mode index modulation is also promising for harsh free space and underwater environments, as demonstrated in [28], [29]. The mode index modulation concept is still limited by how many beams can be generated by the transmitter, the highest beam order that can be collected by the receiving optics, and the maximum number of shapes that can be correctly distinguished by the used ML algorithm.

Many studies based on the use of OAMs to communicate in free space, optical fibers, and underwater optical communication have been reported [7], [8]. The OAM degree of freedom has also shown to be promising in radio frequency communications. What follows is a brief summary of the use of OAM in each of these communication mediums.

\section{A. OAM Communications in ...}

1) Free Space: FSO is a license-free technology that provides line-of-sight communication between two terminals connected via laser beams propagating in the atmosphere. FSO is a solution to 'last mile' and 'last meter' problems in communication networks, especially when optical fiber connectivity is scarce. Gibson et al. demonstrated in 2004 that OAM beams can be used to carry information in free space [30]. Eight years after this seminal experiment, beyond 1 Tbps FSO communication over a short free-space distance was reported in [31]. The first Tbps scale demonstration opened the doors to several followup high-speed reports beyond 100 Tbps [32] and also exceeding 1 Pbps (that is a 1000 Tbps) over laboratory test-benches [33]. An outdoor demonstration reported a $400 \mathrm{Gbps}$ free space transmission over a distance of $120 \mathrm{~m}$ using 4 OAM modes, each carrying a 100 Gbps signal [34]. OAM beams have also been suggested to increase the capacity of unmanned aerial vehicles (UAVs) FSO communication [35]. Authors of [35] successfully demonstrate a two-OAM beam transmission, each carrying a 40 Gbps data signal, over the same UAV-ground station link. 
2) Optical Fiber: For several years, increasing the transmission capacity of optical fiber communication links has been ensured using wavelength division multiplexing (WDM) and polarization division multiplexing (PDM). The former consists of encoding independent data streams over different wavelength channels, which are then propagated over the same optical fiber. The latter is based on the use of two orthogonal polarization states to transport two independent signals over the same fiber. With the continuous demand for more and more capacity, space division multiplexing (SDM) was proposed to solve the bandwidth bottleneck in fiber communication [36]. SDM has two approaches based on the use of two different optical fiber types; fibers that support multiple spatial modes and fibers have multiple cores. Multicore fibers (MCFs) can be viewed as a superposition of single mode fibers (SMFs) sharing the same outer cladding. SDM in fibers supporting a set of spatial modes is known as SDM over few mode fibers (FMFs). The idea of using multiple modes of light over the same fibers dates back to the 1980s with mode division multiplexing (MDM) in multimode fibers (MMFs) [37], [38], but was objected at that time because of a major performance-limiting factor, which is intermodal dispersion in particular with fibers supporting a few hundreds of modes. The idea was then resurfaced with fibers supporting a manageable number of modes (i.e., FMFs). OAM was proposed as a potential mode of choice for SDM over FMFs. The first experiment on the topic was conducted by Bozinovic et al. [39] and reported a 1.6 Tbps transmission using 2 OAM states and ten wavelength channels over a $1.1 \mathrm{~km}$ specially designed fiber, known as "vortex fiber".

Unlike linearly polarized (LP) modes, which are widely used in SDM, OAM does not require digital signal processing (DSP) operations to separate between the beams after propagation through the fibers [40]. This is a convenient feature to ensure convergence between free space optics and fiber technologies. Modes propagating in free space can be coupled to optical fibers and possibly propagated to free space without any intermediate conversion devices to convert the information from OAM to LP modes and then to OAM modes again. Another potential for OAM beams is the ability to propagate in MMFs with minimum crosstalk over distances of a few kilometers [41]. This gives another clear advantage of OAMs compared to LP modes, which severely suffer from crosstalk when propagating through MMFs.

3) Underwater: Over the last few years, UWOC has seen tremendous progress for short-range transmissions of a few tens of meters through the water [42]. UWOC is proposed to cope with the limitations of the acoustic communication technology suffering from low latency and limited bandwidth in order to satisfy the growing demand for higher data rates for a wide range of applications, including marine life exploration, climate change control, and oil pipeline monitoring. Because of UWOC, Gbps transmission rates are now possible. UWOC mainly benefits from the blue-green $(400-500 \mathrm{~nm})$ region of the spectrum due to the low attenuation of light signals in this window and the recent advances in the development of energy-efficient light-emitting diodes and lasers. OAM was proposed to increase the capacity of UWOC further and achieve multi-gigabit links [43], [44]. A 3 Gbps transmission over a 2.96 $\mathrm{m}$ long water tube using two OAM beams generated using homemade SPPs was reported [43]. Up to $40 \mathrm{Gbps}$ transmission was reported using four green OAM states through a $1.2 \mathrm{~m}$ of water [44]. Very recently, it has been shown in [45] that OAM beams can propagate over a distance of $55 \mathrm{~m}$ underwater channel.

4) Radio: In 2007, it was shown, through simulations by Thidé et al., that OAM-carrying waves can be generated in the RF domain [46]. A seminal experiment reporting the transfer of information over a radio OAM beam was conducted in Venice (Italy) [47]. This demonstration was followed by a millimeter-wave transmission reporting a total data rate of 32 Gbps over a distance of $2.5 \mathrm{~m}$ [48]. Many studies in the literature have investigated how to optimize the design of OAM-based RF systems, particularly in transmitting and receiving antennas designs. RF radio waves can be generated using SPPs in a similar manner to the optical waves or using uniform circular antenna (UCA) arrays [49]. UCAs are the most common generation tool of radio OAMs. A UCA is composed of a set of $N$ elements that are equally spaced around the circumference, as can be seen in Fig. 10. A phase shift of $\Delta \phi=2 \pi \ell / N$ is set 


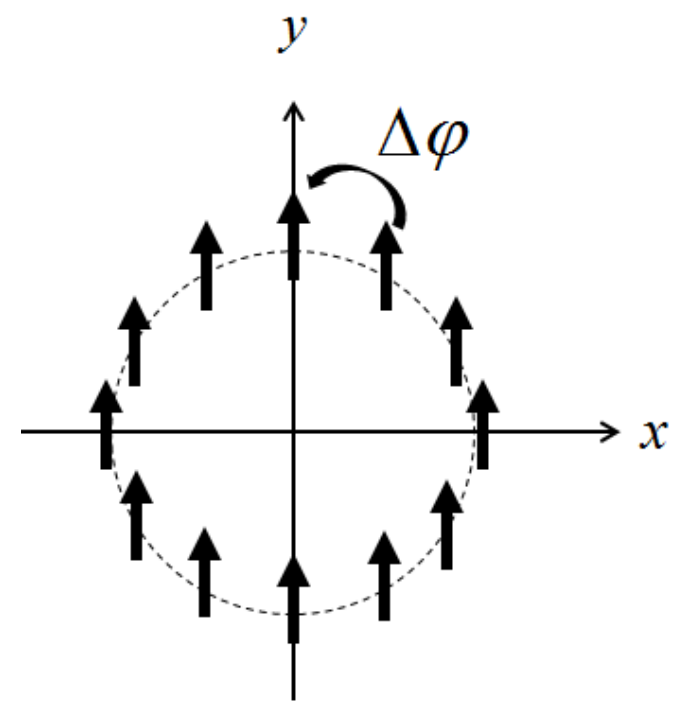

Fig. 10. An illustration of an $N$ element UCA, with an azimuthal phase difference between two adjacent elements of $\Delta \phi=2 \pi \ell / N$.

between every two adjacent elements, where $\ell$ is the desired OAM order to generate. Note that the same UCA can be used to generate multiple radio OAM, and the maximum order is $|\ell| \leq \frac{N}{2}-1$. UCAs can also be used for the detection of radio OAM beams [50].

\section{B. OAM Challenges in Communication}

Although OAM multiplexing holds great potentials in various communication technologies, the deployment of OAM communication links in different propagation media is subject to several challenges, including divergence and the non-resilience to propagation effects. In the following, we briefly discuss some of those challenges.

1) Divergence: As it propagates in free space, light beams tend to diverge. For a Gaussian beam (approximate output beam shape of most of the lasers), the beam varies along the propagation direction as described by Eq. (9). The divergence of OAM beams scales with $\sqrt{|\ell|+1}[51]$, which imposes a huge requirement for the design of receiving apertures needed to collect a beam at the receiver compared to the one necessary for a Gaussian beam collection. Similar to optical communication, divergence is a significant challenge for RF OAM and might result in bulky receiving antennas design.

2) Turbulence for Optical Wireless Links: Turbulence in optical wireless communication has been a severe obstacle for the wide-scale deployment of OAM communication (and indeed any higher order modes [52]). Propagation through turbulent channels leads to an exchange of energy between co-propagating OAM beams [53], known as intermodal crosstalk. An illustration of an OAM beam propagating through atmospheric turbulence and the resulting intermodal crosstalk is depicted in Fig. 11. Intermodal crosstalk is deleterious to optical wireless communication and results in a mode-dependent loss. Turbulence in free space originates from the random refractive index fluctuations caused by the atmosphere's temperature and pressure variations. Turbulence in an underwater environment is caused by random fluctuations of temperature and salinity along the propagation channel. Air-bubbles impose additional challenges by partially obstructing or completely the propagating beams.

3) Multipath for RF links: RF OAM links are subject to an effect known as multipath fading that could lead to severe performance degradation. Multipath is an effect in which the signal sent by the transmitter arrives in multiple paths to the receiver and is mainly caused by reflections from surfaces 
(a)

(b)

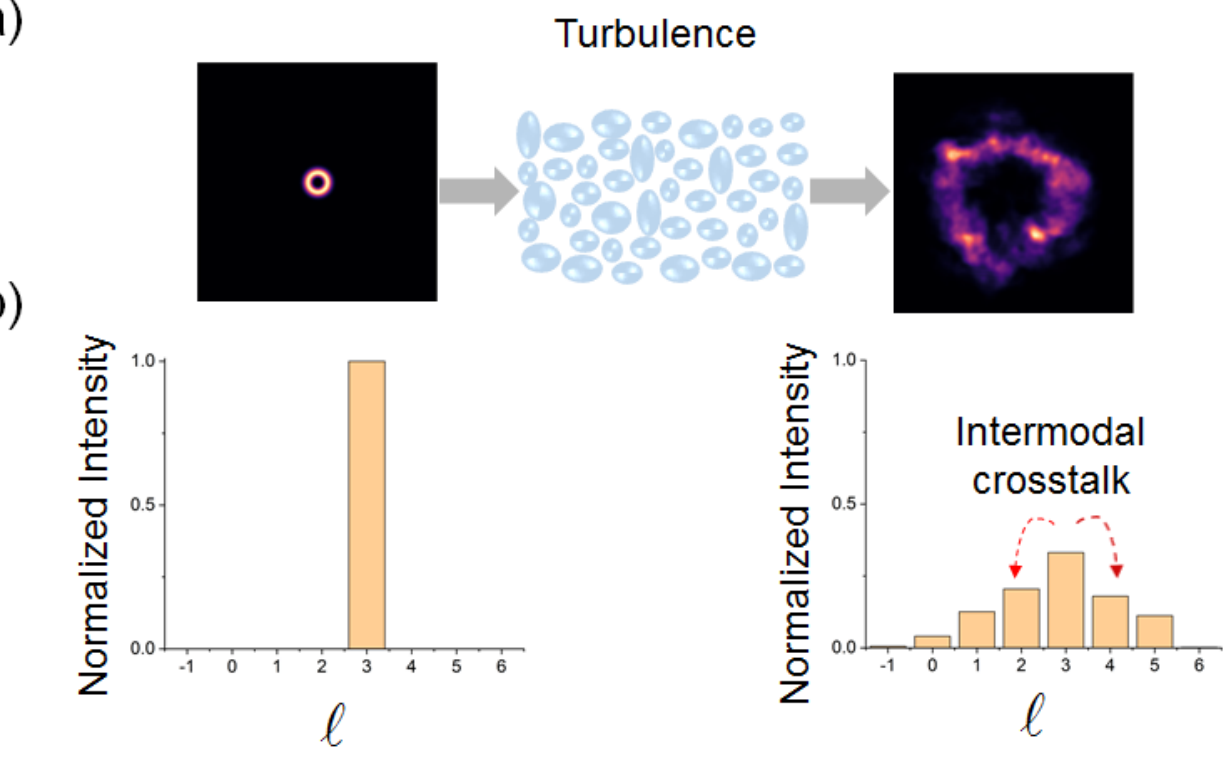

Fig. 11. An illustration of (a) OAM mode waterfront distortion after $1 \mathrm{~km}$ of propagation through a turbulent atmosphere (b) intermodal crosstalk caused by turbulence. The turbulence is simulated using the modified von kármán turbulence model. More details on atmospheric turbulence modeling and simulations can be found in [54], and references therein.

along the propagation path (e.g., ground, water, buildings) (and/or atmospheric effects). Multipath results in intra-and inter-channel crosstalk, i.e., energy from one OAM beam is coupled into the same beam and also to other OAM beams [55]. Multipath also leads to intersymbol interference (ISI) that occurs when the time delay between the propagation paths is comparable with the signal duration.

4) Bulky Generation \& Detection Devices: Several generation and detection methods have been proven to be efficient to generate high-purity beams and detect beams with high accuracy, as shown in Section III] However, many of these techniques require maintaining fine alignment and can be bulky for system deployment in practical scenarios. For example, despite their versatility, SLMs are bulky and require maintaining alignment with the laser and also blocking the unwanted diffraction orders.

\section{OAM Communication Perspectives}

1) Beating the Effect of Turbulence: Beating the effect of turbulence can be done using several techniques such as adaptive optics (AO) and digital signal processing techniques. AO is a technology that aims to measure the aberrations caused by random propagation effects at the beam level using a wavefront sensor and correct for them using a wavefront corrector. AO can be installed in two different schemes; at the receiver or the transmitter:

- Pre-compensation scheme: when the AO is installed at the transmitter, and within this scheme, the beam is distorted before propagation. The distortion is compensated along the propagation, and a quasi-perfect beam is obtained at the transmitter.

- Post-compensation scheme: when the AO is installed at the receiver, the received distorted beam is corrected before being decoded.

Both AO schemes have been used to correct for turbulence in OAM multiplexing high-speed FSO experiments [56].

Compensating for turbulence effects using DSP-based solutions involves coding at the transmitter or estimation techniques at the receiver. Beamforming techniques have been equally demonstrated. The idea is to encode a superposition of beams, and the pre-generated crosstalk is compensated by the channel 
effects [57]. The major requirements of this technique are the continuous feedback from the receiver and the accurate phase estimation at the transmitter. Similarly, the choice of modes at the transmitter also makes a significant difference in the performance of a system through turbulence [58]-[60].

Another relatively new technique to cope with the effect of turbulence is modal diversity. Modal diversity harnesses the hypothesis that different modes experience turbulence slightly differently when they propagate - although this is still unproven and not modeled [61], [62]. In a single-channel communication system, there is a certain probability of error. If two or more statistically independent channels are used, the error probabilities can be multiplied and are therefore dramatically reduced. Modal diversity has been demonstrated using different OAM modes, which also have different sizes and divergences as they propagate [63], [64], as well as a combination of HG and LG modes with identical sizes [61].

2) Coping with OAM RF Challenges: To cope with the impact of divergence in RF OAM links, particularly over long distances, a time-domain OAM reception technique was proposed in [65]. The idea is to use a single antenna to detect multiple OAM beams in the time domain rather than the space domain by rotating the OAM at the transmitter. This can considerably ease the requirement to collect the full OAM ring to detect the encoded information sent by the transmitter. To minimize the ISI in RF OAM links, authors of [66] proposed using the orthogonal frequency division multiplexing (OFDM) $]^{1}$ technique to encode the digital signals on the OAM beams.

3) Toward the Use of Complete Modal Bases: Using the full Laguerre Gaussian mode basis has been found to be efficient in communication [67]. More than 100 LG modes over 3 wavelengths were successfully multiplexed and de-multiplexed using $\mathrm{CGHs}$, demonstrating the feasibility to encode information using the full LG basis [67]. The use of 4 LG modes with different radial and azimuthal indices enabled an FSO transmission of 400 Gbps [68].

There have been a number of studies into the resilience of different mode sets to turbulence. HG modes have been shown to be more robust than OAM and LG modes because certain HG modes have a resilience to lateral translation (tip and tilt) at the detector [59], [69]. Modal diversity has also been demonstrated with identical order LG and HG modes, which have the same size and divergence properties, indicating that the diversity gain comes from the mode shape and not just its size [61]. This has subsequently been demonstrated at high speed [70].

4) Toward Small-Footprint Mode Generation/Detection Devices: Compact twisted light transmitters that could be tuned at the nano-second speed have been demonstrated [71]. Recently a tunable OAM microlaser able to switch between up to 5 OAM states has been demonstrated [72]. Still, such a laser hasn't been tested for a communication experiment. Microstructured SPPs that could be integrated on top of lasers have also been constructed using two-photon lithography (TPL) 2 and used to establish FSO communication at the near-infrared region [73].

Small foot-print detection techniques have also been demonstrated [74], [75]. Authors of [74] reported the fabrication (using the TPL technique) of a micro-scale mode sorter capable of detecting OAMs with $|\ell| \leq 3$ over a broad wavelength range. A miniaturized OAM mode sorter fabricated using electron beam lithography ${ }^{3}$ was demonstrated in [75].

These efforts on miniaturizing OAM mode generation and detection techniques are a significant step toward using OAM in real-life applications. In addition to generation and detection devices, particular efforts should be equally given to the design of OAM amplifiers.

\footnotetext{
${ }^{1}$ OFDM is a digital modulation technique widely used in wireless radio communication and consists of the use of multiple spaced sub-carriers instead of a single carrier frequency, which considerably lowers ISI

${ }^{2} \mathrm{~A} 3 \mathrm{D}$ printing fabrication technique with a $100 \mathrm{~nm}$ resolution based on two-photon absorption to create features in a photosensitive material known us photoresist or resist.

${ }^{3} \mathrm{~A}$ 3D printing technique with sub-10 $\mathrm{nm}$ resolution that is based on scanning a focused beam of electrons on a surface covered with a resist.
} 

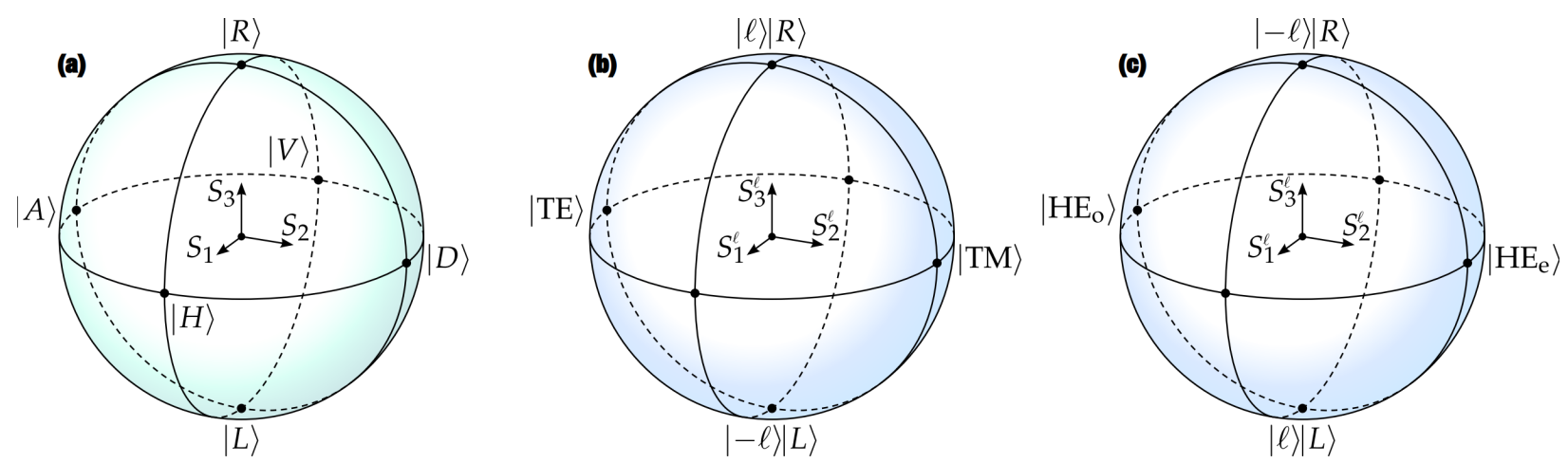

Fig. 12. (a) The Poincaré sphere showing all the possible states of polarization of light indicated in Dirac notation with the Stokes parameters $\left(S_{1,2,3}\right)$ also visualised. Vector vortex modes can be visualised on a Higher-Order Poincaré sphere shown in (b) and (c), with the definitions of the labels given in Sec. V

\section{VECTOR VORTEX BEAMS}

So far, the discussion has been limited to scalar spatial modes or modes with uniform polarization. Scalar modes are, in fact, a special case of the Helmholtz equation in Eq. (1). There is another more general class of modes with non-uniform polarization, which are called "vector" modes [76]-[79].

Before we delve further into what a vector mode is, a brief recap on polarization is necessary. Polarization is a property of transverse waves, such as a laser beam, which specifies the orientation of the oscillations. Since light is an electromagnetic wave with both electric and magnetic field components, we define the polarization of light as the orientation of the electric field. In a quantum sense, when considering individual photons, the polarization of the light is determined by the spin of the photons.

Stemming from this quantum spin, there are only two possible polarizations: left and right circular polarization. Linearly polarized light consists of a superposition of the two circular polarization states. The state of polarization of a laser beam can be visualized on a so-called Poincaré sphere, shown in Fig. 12(a). Any point on the surface of the sphere is a valid polarization state, with the north and south poles being the two circular polarization states. If one moves down one of the longitudes of the sphere to the equator, the light becomes linearly polarized, namely horizontal, vertical, diagonal, or anti-diagonal, or somewhere in between. If the state of the polarization is between linear and circular, we call this elliptical polarization.

A mathematical way of describing the state of polarization of light (or electromagnetic radiation in general) is via Stokes parameters, which are indicated in Fig. 12 as $S_{1}, S_{2}$ and $S_{3}$ and described in more detail in Sec. V-B1] [80]. The Stokes parameters are Cartesian coordinates that can be easily mapped to spherical coordinates with the radius of the sphere being the intensity, $S_{0}=I$, of the wave.

Since we can represent an arbitrary state of polarization using a superposition of left and right polarization components, which form an orthonormal basis, we can describe a unit-amplitude, paraxial vector fields (or vector modes) in the form

$$
\mathbf{U}(\mathbf{s})=\cos (\theta) U_{L}(\mathbf{s}) \hat{e}_{L}+e^{i \alpha} \sin (\theta) U_{R}(\mathbf{s}) \hat{e}_{R},
$$

where $\mathbf{s}$ refers to the field's transverse coordinates which can be Cartesian where $\mathbf{s} \triangleq(x, y)$ or cylindrical where $\mathbf{s} \triangleq(r, \phi)$. The orthogonal left and right circularly polarized unit vectors are given by $\hat{e}_{L}$ and $\hat{e}_{R}$, respectively. The amplitude factors $\cos (\theta)$ and $\sin (\theta)$ allow a smooth transition of the field from pure scalar (when $\theta=0$ or $\pi / 2$ ) to pure vector (when $\theta=\pi / 4$ ) given that $\theta \in[0, \pi / 2]$. For generality, $\alpha$ is a phase difference between the two components of the field. Finally, $U_{L}(\mathbf{s})$ and $U_{R}(\mathbf{s})$ are the spatial electric field solutions, such as those given earlier in Sec. II. 

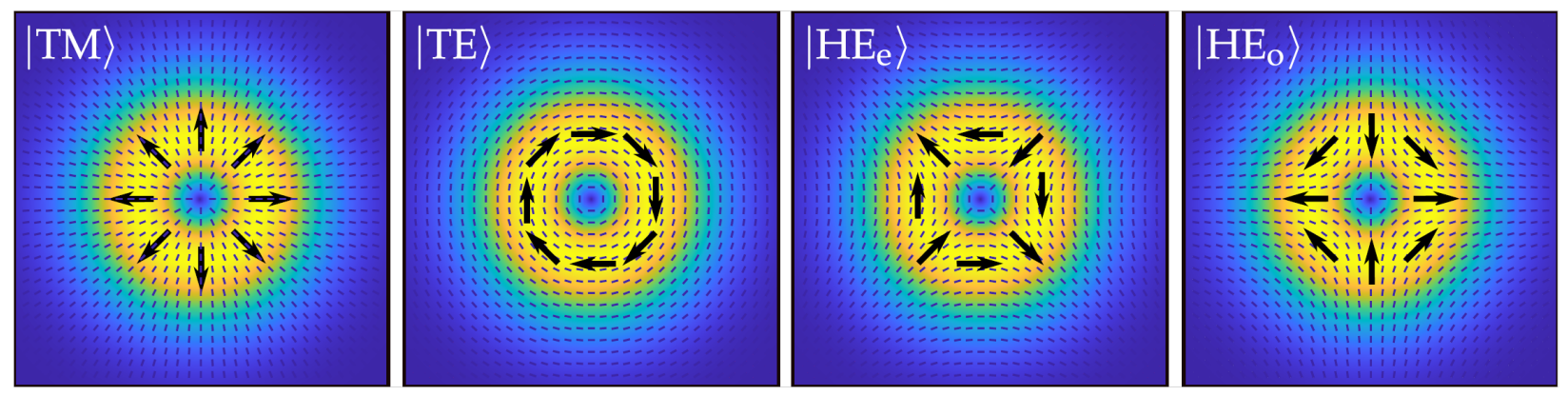

Fig. 13. The four cylindrical vector vortex modes showing intensity and polarization direction.

A common yet special case that has received a lot of attention is the so-called cylindrical vector vortex $(\mathrm{CVV})$ modes, which are colloquially OAM vector beams. These can be written in cylindrical coordinates with $\mathbf{s} \triangleq(r, \phi)$, as

$$
\mathbf{U}(\mathbf{s})=\cos (\theta) A(r) e^{i \ell \phi} \hat{e}_{L}+e^{i \alpha} \sin (\theta) A(r) e^{-i \ell \phi} \hat{e}_{R}
$$

where $A(r) \exp ( \pm i \ell \phi)$ describes a scalar OAM mode with azimuthal index $\ell$. These CVV modes are non-separable states of light where OAM and polarization are coupled. By adjusting the parameters $\theta$ and $\alpha$ we can construct all the states on the higher-order Poincaré sphere, which is shown in Fig. 12 b) and (c) for $\ell= \pm 1[81]$.

A convenient notation exists whereby we can write vector modes in a clear and concise manner called Dirac (or bra-ket) notation [82], [83]. Dirac notation is a tool borrowed from the quantum world, which will be described in more detail in Sec. VI. Using this notation, Eq. 26 simply becomes

$$
|U\rangle=\cos (\theta)|\ell\rangle|L\rangle+e^{i \alpha} \sin (\theta)|-\ell\rangle|R\rangle
$$

From this, there are four cylindrical vector vortex mode bases given by

$$
\begin{aligned}
|\mathrm{TM}\rangle & =\frac{1}{\sqrt{2}}(|-\ell\rangle|L\rangle+|\ell\rangle|R\rangle), \\
|\mathrm{TE}\rangle & =\frac{i}{\sqrt{2}}(|-\ell\rangle|L\rangle-|\ell\rangle|R\rangle), \\
\left|\mathrm{HE}_{\mathrm{e}}\right\rangle & =\frac{1}{\sqrt{2}}(|\ell\rangle|L\rangle+|-\ell\rangle|R\rangle), \\
\left|\mathrm{HE}_{\mathrm{o}}\right\rangle & =\frac{i}{\sqrt{2}}(|\ell\rangle|L\rangle-|-\ell\rangle|R\rangle) .
\end{aligned}
$$

These are known as the Transverse Magnetic (TM), Transverse Electric (TE), Hybrid Electric Even (HE $\mathrm{H}_{\mathrm{e}}$, and Hybrid Electric Odd $\left(\mathrm{HE}_{\mathrm{o}}\right)$ modes and are visualized in Fig. 13. These modes have become a topic of great interest because of the higher dimensional encoding capabilities for mode division multiplexing in classical optical communications as well as quantum systems discussed in more detail in Sec. VI-D.

In classical communications, it has been claimed that the use of vector vortex modes would make a system more resilient to atmospheric turbulence (and hence more reliable) [84]-[86], but it was found that this may not be the case [60]. The polarization distribution of these modes is similar to the fiber LP modes, which makes hybrid fiber to free-space systems a possibility [87].

Other uses of vector modes include optical trapping [88]-[90], due to the tight focus-ability and hence trapping efficiency of vector vortex beams, micro-drilling and material processing [91]-[94], particle acceleration [95] and even in microscopy [96], [97]. 


\section{A. Generation using Spatial Light Modulators}

A number of methods for the creation of vector modes have arisen over the years. Passive devices such as meta-materials and q-plates are able to do so in a static fashion, but often in a lab environment, these methods are too limiting. One of the first methods capable of creating vector vortex modes was to use a Sagnac interferometer arrangement built around a spatial light modulator as one of the mirrors and a dove prism to add a phase shift [98]. Subsequently, more versatile approaches have arisen.

In a lab environment, liquid crystal spatial light modulators, which are, in essence, digital holograms, are ubiquitous for beam shaping [99]. Unfortunately, they are typically only able to modulate light with a specific linear polarization. As such, if they are to be used to create vector modes, then the output beams must somehow be separated and passed through wave plates (phase retarders) to impose different polarizations on each mode before coherently recombining them into a vector mode. High resolution digital micro-mirror devices are becoming popular as they can also be used as digital holograms, albeit with lower light efficiency, but for arbitrary polarization [100]. Another benefit of DMDs is their high speed compared to liquid crystal spatial light modulators [19], [24], [101].

Here, two methods will be described to create arbitrary vector modes. The first method makes use of a DMD and is shown in Fig. 14(a) [102]. An incoming, diagonally polarized beam is split using a Wollaston prism into horizontal and vertical components that symmetrically diverge at a small angle (approximately $1^{\circ}$ ). A $4 f$ telescope arrangement maps these diverging components to a DMD, after which the zero-order reflections will continue to separate. A quarter-wave plate (QWP) is placed into the path of these two beams to convert the opposite linear polarization states into left and right circular polarized beams. The DMD displays a binary hologram, which is the superposition of two holograms, one intended for each beam. The gratings of the holograms are rotated and adjusted, such that the resulting modulated first-orders emerge from the DMD overlapped as a vector beam. A spatial filter is used to separate this beam from the reflected zero-orders. In this arrangement, it is also possible to use the DMD as a phase retarder to further modify the polarization of the component beams (for example, to reduce the vector quality factor, see Sec. V-B2 . If a Wollaston prism is not available, it is possible to use a standard polarizing beam splitter (PBS); however, the setup will be significantly larger.

The second method uses a liquid crystal spatial light modulator and relies on a Sagnac interferometer arrangement to overlap the spatially modulated beams, shown in Fig. 14 (b) [103]. An incoming horizontally polarized, expanded beam reflects off an SLM, which has two holograms side-by-side intended for each polarization component of the beam. The grating of each hologram is configured such that the first orders are overlapped at the spatial filter after the SLM. After this spatial filter, each half of the beam will be spatially separated by a certain small distance. A half-wave plate (HWP) is used to rotate the polarization of these two beam components to diagonal. In order to overlap these two parallel beams, a Sagnac interferometer is used with a polarizing beam splitter. The interferometer should be adjusted so that one of the horizontal and one of the vertical components overlap, after which a spatial filter is used to block the non-overlapping beams. Finally, the beam, which is a vector mode with horizontal and vertical polarization, is passed through a QWP to convert it to a more standard left and right circular polarization.

\section{B. Characterisation of Vector Vortex Beams}

The tools used to characterize scalar modes such as OAM modes are usually quite straight forward, as discussed in Sec. III. However, because vector modes also have a polarization component, it is important to include that in their analysis.

Several techniques exist for "sorting" and detecting vector vortex modes, for example those given in Refs. [60], [104]-[107]. These techniques are useful when the orthogonality of the modes in a system is harnessed, such as in multiplexed optical communications, however, here we focus more generally on the characterisation of vector beams. In this section, we describe two main techniques used to measure the 
(a)
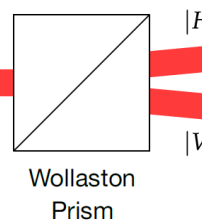

$|V\rangle$

QWP

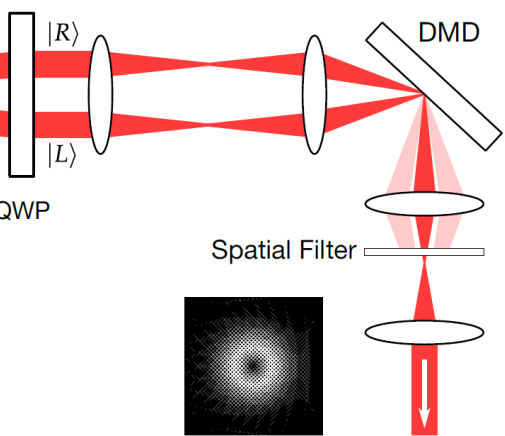

(b)

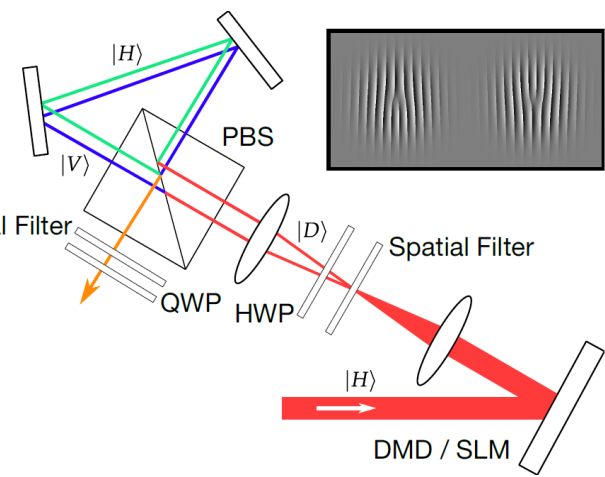

Fig. 14. Two versatile experimental setups for the creation of arbitrary vector beams with insets of example holograms. A digital micromirror device (DMD) is used in (a) to modulate two different polarization incoming beams using two superimposed holograms with the resulting first-orders overlapped [102]. In (b), a spatial light modulator is split into two holograms and the first-orders of each hologram are overlapped with a Sagnac interferometer arrangement [103].

spatial distribution of polarization of a beam, called Stokes polarimetry, and the so-called Vector Quality Factor (VQF), which is a measure of the degree of non-separability of the vector mode.

1) Stokes Polarimetry: Stokes polarimetry is a mature technique in optics used to measure the spatial polarization structure of an optical field [80]. This is a useful tool for measuring vector modes as it allows us to visualize the polarization orientation and ellipticity across the beam. One can then verify whether this distribution corresponds to what is expected for the mode of interest.

As mentioned before, the state of polarization of light can be fully characterised with four Stokes parameters, $S_{0}$ through $S_{3}$. The first parameter, $S_{0}$ describes the total intensity of the optical field. The remaining three parameters describe the polarization. $S_{1}$ represents the amount of linear polarization (horizontal and vertical), $S_{2}$ the amount of diagonal polarization $\left(45^{\circ}\right.$ and $\left.135^{\circ}\right)$ and $S_{3}$ the amount of circular polarization (left and right). The Stokes parameters can conveniently be measured as the corresponding intensities of the light after it has been filtered using polarizers and phase retarders [108]. In summary, each parameter is given by

$$
\begin{aligned}
& S_{0}=I_{H}+I_{V}=I_{0}, \\
& S_{1}=I_{H}-I_{V}=2 I_{H}-S_{0}, \\
& S_{2}=I_{D}-I_{A}=2 I_{D}-S_{0}, \\
& S_{3}=I_{R}-I_{L}=2 I_{R}-S_{0},
\end{aligned}
$$

where $I_{x}$ are the horizontal, vertical, diagonal, anti-diagonal, left, and right polarization intensity components. Notice that not all the measurements are required because we can rearrange the equations, knowing the relationship between the various polarization states.

By measuring these parameters in a spatially discrete manner, for example, with a camera, we can plot the polarization ellipse at multiple spatial points, depending on the required resolution. The polarization ellipse is an intuitive way to visualize polarization in two dimensions as opposed to the Poincare sphere. At the extremes, for linear, the ellipse is stretched into a line pointing in the direction of polarization, and for circular polarization, the ellipse is round. Fig. 15. a) shows a parameterised ellipse and an example of a spatial polarization map in Fig. 15 (b) of the cylindrical vector vortex modes in Fig. 13, with the parameters given by [103]

$$
A=\sqrt{\frac{1}{2}\left(S_{0}+\sqrt{S_{1}^{2}+S_{2}^{2}}\right)},
$$



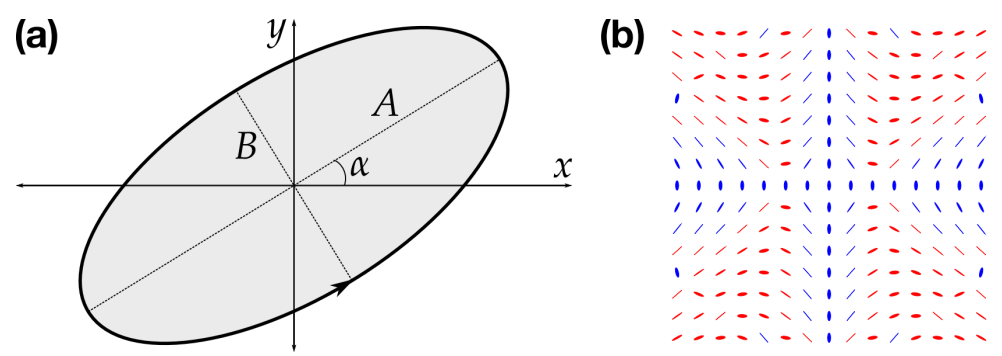

Fig. 15. The polarization ellipse, which represents the state of polarization of an optical field. Equations 30 , 31 and 32 give the parameters in (a). The arrow indicates the direction of polarization but may be visualised differently, such as in red and blue for left and right, depending on the application with an arbitrary example in (b).

TABLE I

THE SPATIAL INTENSITY MEASUREMENTS USED FOR THE VQF.

\begin{tabular}{c|cccccc}
\hline Polarization & $\ell=\mathbf{1}$ & $\ell=-\mathbf{1}$ & $\alpha=\mathbf{0}$ & $\alpha=\pi / \mathbf{2}$ & $\alpha=\pi$ & $\alpha=\mathbf{3} \pi / \mathbf{2}$ \\
\hline $\mathrm{L}$ & $I_{11}$ & $I_{12}$ & $I_{13}$ & $I_{14}$ & $I_{15}$ & $I_{16}$ \\
$\mathrm{R}$ & $I_{21}$ & $I_{22}$ & $I_{23}$ & $I_{24}$ & $I_{25}$ & $I_{26}$ \\
\hline$B=\sqrt{\frac{1}{2}\left(S_{0}-\sqrt{S_{1}^{2}+S_{2}^{2}}\right)}$ \\
$\alpha=\frac{1}{2} \arctan \left(S_{2} / S_{1}\right)$.
\end{tabular}

2) Vector Quality Factor: The vector quality factor (VQF) provides a convenient metric that indicates the degree of coupling between the polarization and spatial degrees of freedom of a vector mode: in other words, the "vectorness" of the measured mode [109], [110]. A value of zero indicates no coupling (i.e., the mode is scalar), and a value of one indicates strong coupling (i.e., the mode is vector).

The VQF borrows tools from the quantum world and exploits the fact that vector beams can be viewed as classically "entangled", or non-separable in their spatial and polarization degrees of freedom. As such, the VQF is a classical measure of the concurrence, $C$, of the beam, given by

$$
\mathrm{VQF}=\Re\{C\}=\Re\left\{\sqrt{1-\sum_{i}\left\langle\sigma_{i}\right\rangle^{2}}\right\},
$$

where $\left\langle\sigma_{1}\right\rangle,\left\langle\sigma_{2}\right\rangle$ and $\left\langle\sigma_{3}\right\rangle$ are the expectation values of the so-called Pauli operators. These values (summarised in Tab. I) can be measured experimentally with a total of twelve spatial intensity measurements, six for each polarization of the beam (left and right circular), to map the higher order Poincare sphere described earlier. The expectation values of the Pauli operators are then calculated using these measurements as follows:

$$
\begin{aligned}
& \left\langle\sigma_{1}\right\rangle=\left(I_{13}+I_{23}\right)+\left(I_{15}+I_{25}\right), \\
& \left\langle\sigma_{2}\right\rangle=\left(I_{14}+I_{24}\right)+\left(I_{16}+I_{26}\right), \\
& \left\langle\sigma_{3}\right\rangle=\left(I_{11}+I_{21}\right)+\left(I_{12}+I_{22}\right) .
\end{aligned}
$$

For each polarization, the six intensity measurements are the modal decomposition of the beam into the pure OAM modes, $e^{i \ell \phi}$ and $e^{-i \ell \phi}$, as well as four super-positions of these modes with varying inter-modal phase given by $\alpha: e^{i \ell \phi}+e^{i \alpha} e^{i \ell \phi}$. Spatial mode bases other than OAM may be used, but here our focus is on cylindrical vector vortex modes.

Fortunately, these measurements can be done simultaneously with a polarization grating, multiplexed holograms and a camera, illustrated in Fig. 16, which also has insets of the various phases required for the spatial measurements. 


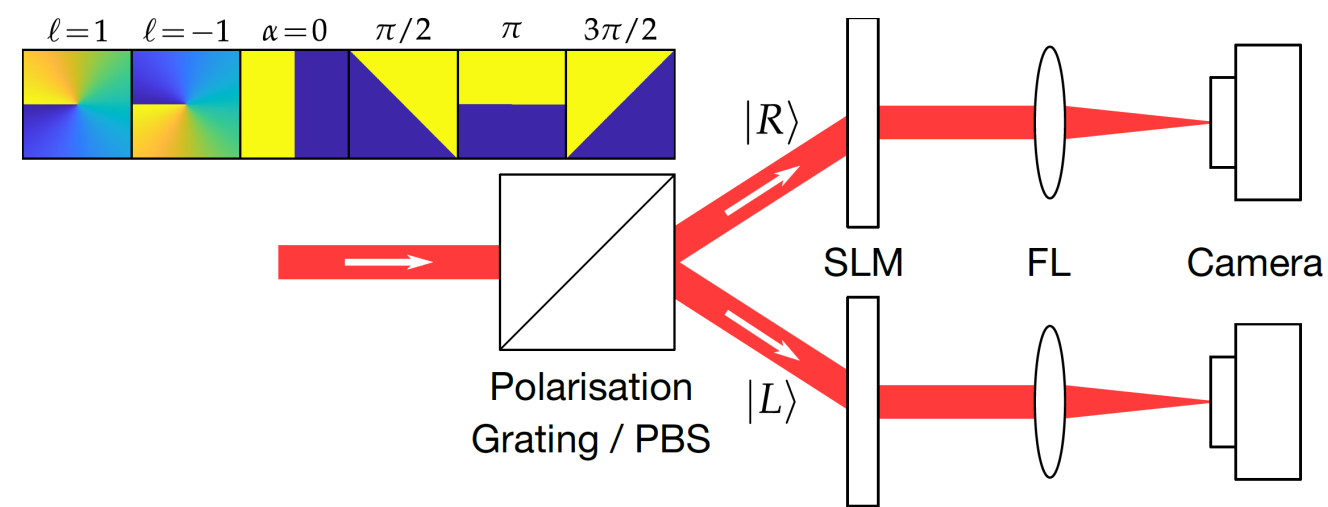

Fig. 16. An example setup to measure the vector quality factor. A polarization grating separates the left and right polarization and a modal decomposition is performed on each using a spatial light modulator (SLM), Fourier lens (FL) and a camera. The insets at the top left are the phases for each of the spatial measurements.

\section{OAM IN QUANTUM OPTICS}

\section{A. Canonical Quantization}

Up to this point, we have described light as an electromagnetic wave phenomena fully described by classical means. Nonetheless, there are some scenarios where the quantum description is more appropriate, i.e., in the case of low intensity or when non-linear processes are involved. A vast literature study such cases [111]-[116], indeed, the quantum theory of light and matter interaction is in the realm of quantum optics.

The first step when studying quantum optics phenomena, is to quantize the electromagnetic field. Within the Coulomb gauge [82], the vector potential $\mathbf{A}$ obeys the wave equation

$$
\nabla^{2} \mathbf{A}-\frac{1}{c^{2}} \frac{\partial^{2} \mathbf{A}}{\partial t^{2}}=0
$$

where $c$ is the speed of light in the vacuum, $\nabla \mathbf{A}=0$, and the electric and magnetic fields are given by

$$
\begin{aligned}
\mathbf{E} & =-\frac{\partial \mathbf{A}}{\partial t}, \\
\mathbf{B} & =\nabla \times \mathbf{A} .
\end{aligned}
$$

Considering periodic boundary conditions in a box of volume $V=L^{3}$, we arrive to the expression

$$
\mathbf{A}(\mathbf{r}, t)=\frac{1}{\sqrt{V}} \sum_{\mathbf{k}, \lambda}\left[c_{\mathbf{k}, \lambda} e^{-i\left(\omega_{k} t-\mathbf{k} \cdot \mathbf{r}\right)}+c_{\mathbf{k}, \lambda}^{*} e^{i\left(\omega_{k} t-\mathbf{k} \cdot \mathbf{r}\right)}\right] \mathbf{e}_{\mathbf{k}, \lambda},
$$

where $c_{\mathbf{k}, \lambda}$ is a complex coefficient, $\omega_{k}=c k$ and $\mathbf{e}_{\mathbf{k}, \lambda}$ corresponds to the polarization vectors. Note that Eq. (38) corresponds to a plane wave expansion and in principle, we can arrive to a similar expression for a different spatial mode.

What follows is a procedure known as canonical quantization. This is done by taking a set of dynamic variables that allows calculating any quantity of the system at any time $t$ (upon the proper time evolution). From the set of dynamic variables, we can pick the ones that come in pairs of canonically conjugate variables, namely the coordinate and momentum $(q, p)$. The idea now is to replace each pair of coordinates $(q, p)$ by a pair of operators $(\hat{q}, \hat{p})$ that do not commute. To do that, we write the classical Hamiltonian

$$
H=\frac{1}{2} \sum_{\mathbf{k}, \lambda}\left(p_{\mathbf{k}, \lambda}^{2}+\omega_{k}^{2} q_{\mathbf{k}, \lambda}^{2}\right),
$$


where $c_{\mathbf{k}, \lambda}=\frac{1}{2 \omega_{k} \sqrt{\epsilon_{0}}}\left(\omega_{\mathbf{k}, \lambda} q_{\mathbf{k}, \lambda}+i p_{\mathbf{k}, \lambda}\right)$, with the time evolution given by $c_{\mathbf{k}, \lambda}(t)=c_{\mathbf{k}, \lambda}(0) e^{-i \omega t}$. Then, we replace the conjugate variables by their corresponding operators that satisfy the commutation relation

$$
\left[\hat{q}_{\mathbf{k}, \lambda}, \hat{p}_{\mathbf{k}, \lambda}\right]=i \hbar \delta_{\mathbf{k}, \mathbf{k}^{\prime}} \delta_{\lambda, \lambda^{\prime}}
$$

where $\delta_{i, j}$ is the Kronecker delta symbol. All these ingredients allow us to finally write the Hamiltonian of the quantized electromagnetic field as

$$
\hat{H}=\sum_{\mathbf{k}, \lambda} \hbar \omega_{k}\left(\hat{a}_{\mathbf{k}, \lambda}^{\dagger} \hat{a}_{\mathbf{k}, \lambda}+\frac{1}{2}\right)=\sum_{\mathbf{k}, \lambda} \hbar \omega_{k}\left(\hat{n}_{\mathbf{k}, \lambda}+\frac{1}{2}\right),
$$

where

$$
\begin{gathered}
\hat{a}_{\mathbf{k}, \lambda}=\frac{1}{\sqrt{2 \hbar \omega_{k}}}\left(\omega_{\mathbf{k}, \lambda} \hat{q}_{\mathbf{k}, \lambda}+i \hat{p}_{\mathbf{k}, \lambda}\right), \\
\hat{a}_{\mathbf{k}, \lambda}^{\dagger}=\frac{1}{\sqrt{2 \hbar \omega_{k}}}\left(\omega_{\mathbf{k}, \lambda} \hat{q}_{\mathbf{k}, \lambda}-i \hat{p}_{\mathbf{k}, \lambda}\right), \\
\hat{n}_{\mathbf{k}, \lambda}=\hat{a}_{\mathbf{k}, \lambda}^{\dagger} \hat{a}_{\mathbf{k}, \lambda},
\end{gathered}
$$

are known as the annihilation, creation and number operators and the following relations hold

$$
\begin{gathered}
{\left[\hat{a}_{\mathbf{k}, \lambda}^{\dagger}, \hat{a}_{\mathbf{k}^{\prime}, \lambda^{\prime}}^{\dagger}\right]=0,} \\
{\left[\hat{a}_{\mathbf{k}, \lambda}, \hat{a}_{\mathbf{k}^{\prime}, \lambda^{\prime}}\right]=0,} \\
{\left[\hat{a}_{\mathbf{k}, \lambda}, \hat{a}_{\mathbf{k}^{\prime}, \lambda^{\prime}}^{\dagger}\right]=\delta_{\mathbf{k}, \mathbf{k}^{\prime}} \delta_{\lambda, \lambda^{\prime}}}
\end{gathered}
$$

Operatively speaking, the action of the previous operators can be summarized as

$$
\begin{gathered}
\hat{a}_{\mathbf{k}, \lambda}\left|n_{\mathbf{k}, \lambda}\right\rangle=\sqrt{n}\left|(n-1)_{\mathbf{k}, \lambda}\right\rangle, \\
\hat{a}_{\mathbf{k}, \lambda}^{\dagger}\left|n_{\mathbf{k}, \lambda}\right\rangle=\sqrt{n+1}\left|(n+1)_{\mathbf{k}, \lambda}\right\rangle, \\
\hat{n}_{\mathbf{k}, \lambda}\left|n_{\mathbf{k}, \lambda}\right\rangle=n\left|n_{\mathbf{k}, \lambda}\right\rangle, \\
\hat{H}|\psi\rangle=E_{n}|\psi\rangle,
\end{gathered}
$$

where $E_{n}=\hbar \omega_{k}\left(n+\frac{1}{2}\right)$ with $n=0,1,2, \ldots$ and the notation $|\cdot\rangle$ represents the usual ket from Dirac's notation [83]. Finally we can calculate the quantized vector potential by

$$
\hat{\mathbf{A}}(\mathbf{r}, t)=\frac{1}{\sqrt{V}} \sum_{\mathbf{k}, \lambda} \sqrt{\frac{\hbar}{2 \omega_{k} \epsilon_{0}}}\left[\hat{a}_{\mathbf{k}, \lambda} e^{-i\left(\omega_{k} t-\mathbf{k} \cdot \mathbf{r}\right)}+\hat{a}_{\mathbf{k}, \lambda}^{\dagger} e^{i\left(\omega_{k} t-\mathbf{k} \cdot \mathbf{r}\right)}\right] \mathbf{e}_{\mathbf{k}, \lambda},
$$

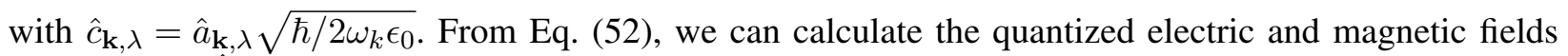
with $\hat{\mathbf{E}}=-\frac{\partial \hat{\mathbf{A}}}{\partial t}$ and $\hat{\mathbf{B}}=\nabla \times \hat{\mathbf{A}}$. 


\section{B. Fock States}

We can generate the state $\left|n_{\mathbf{k}, \lambda}\right\rangle$ by applying $n$ times the creation operator and normalizing

$$
\left|n_{\mathbf{k}, \lambda}\right\rangle=\frac{\left(\hat{a}_{\mathbf{k}, \lambda}^{\dagger}\right)^{n}}{\sqrt{n_{\mathbf{k}, \lambda} !}}|0\rangle
$$

such states are known as Fock states (or number estates) [117] and form a complete orthonormal basis

$$
\left\langle n \mid n^{\prime}\right\rangle=\delta_{n, n^{\prime}}
$$

To exemplify, let us write a single-photon state in the mode $(\mathbf{k}, \lambda)$, that is $\left|1_{\mathbf{k}, \lambda}\right\rangle$. We can further express a two-mode or multimode state as

$$
\begin{gathered}
|\phi\rangle=\sum_{n_{1}, n_{2}}\left|n_{1}, n_{2}\right\rangle, \\
|\varphi\rangle=\sum_{n_{1}, n_{2}, n_{3}, \ldots}\left|n_{1}, n_{2}, n_{3}, \ldots\right\rangle .
\end{gathered}
$$

The quantized electromagnetic field up to this point considers discrete wavelengths and wave vectors. The transition to the continuum can be performed by doing

$$
\frac{1}{\sqrt{V}} \sum_{\mathbf{k}, \lambda}(\ldots) \stackrel{L \rightarrow \infty}{\longrightarrow} \frac{1}{\sqrt{(2 \pi)^{3}}} \int d^{3} \mathbf{k} \sum_{\lambda}(\ldots) .
$$

For Fock states, one can prove that $\langle n|\hat{\mathbf{E}}| n\rangle$ is zero, whereas $\left\langle n\left|\hat{\mathbf{E}}^{2}\right| n\right\rangle$ does not vanish and is proportional to the average detected intensity.

\section{Photons carrying OAM}

We can repeat a similar procedure for the quantization of the electromagnetic field, but this time, in the context of paraxial light. The motivation to do so is to study paraxial modes, in particular the LG modes discussed in the previous sections. In the paraxial regime, the quantization of the electromagnetic field in the continuum limit yields to

$$
\hat{\mathbf{A}}(\mathbf{r}, t)=\int_{k_{0}} d k_{0} \int_{\mathbf{q}} d^{2} q \sum_{\lambda} \sqrt{\frac{\hbar}{16 \pi^{3} \omega_{k} \epsilon_{0}}}\left[\hat{a}_{\mathbf{k}, \lambda} e^{i k_{0} z} e^{-i\left(\theta^{2} z-\mathbf{k} \cdot \mathbf{r}_{\perp}\right)}+\hat{a}_{\mathbf{k}, \lambda}^{\dagger} e^{-i k_{0} z} e^{i\left(\theta^{2} z-\mathbf{k} \cdot \mathbf{r}_{\perp}\right)}\right] \mathbf{e}_{\mathbf{k}, \lambda} .
$$

For the particular case of a LG mode in the paraxial regime, we obtain [82], [116]

$$
\hat{\mathbf{A}}(\mathbf{r}, t)=\int_{k_{0}} d k_{0} \sum_{\lambda, \ell, p} \sqrt{\frac{\hbar}{16 \pi^{3} \omega_{k} \epsilon_{0}}}\left[\hat{a}_{k_{0}, \lambda, \ell, p} e^{i k_{0} z} \mathbf{L G}_{\ell, p}\left(\mathbf{r}_{\perp}, z ; k_{0}\right)+\hat{a}_{k_{0}, \lambda, \ell, p}^{\dagger} e^{-i k_{0} z} L G_{\ell, p}^{*}\left(\mathbf{r}_{\perp}, z ; k_{0}\right)\right] \mathbf{e}_{\mathbf{k}, \lambda},
$$

where

$$
\hat{a}_{k_{0}, \lambda, \ell, p}=\int \hat{a}_{\mathbf{k}, \lambda} \mathcal{L} \mathcal{G}_{\ell, p}^{*}(\mathbf{q}) d^{2} q
$$

is the corresponding annihilation operator for LG modes. The following commutation relations hold

$$
\begin{gathered}
{\left[\hat{a}_{k_{0}, \lambda, \ell, p}^{\dagger}, \hat{a}_{k_{0}^{\prime}, \lambda^{\prime}, \ell^{\prime}, p^{\prime}}^{\dagger}\right]=0,} \\
{\left[\hat{a}_{k_{0}, \lambda, \ell, p}, \hat{a}_{k_{0}^{\prime}, \lambda^{\prime}, \ell^{\prime}, p}^{\prime}\right]=0,} \\
{\left[\hat{a}_{k_{0}, \lambda, \ell, p}, \hat{a}_{k_{0}^{\prime}, \lambda^{\prime}, \ell^{\prime}, p^{\prime}}^{\dagger}\right]=\delta_{\lambda, \lambda^{\prime}} \delta_{\ell, \ell^{\prime}} \delta_{p, p^{\prime}} \delta\left(k_{0}-k_{0}^{\prime}\right) .}
\end{gathered}
$$




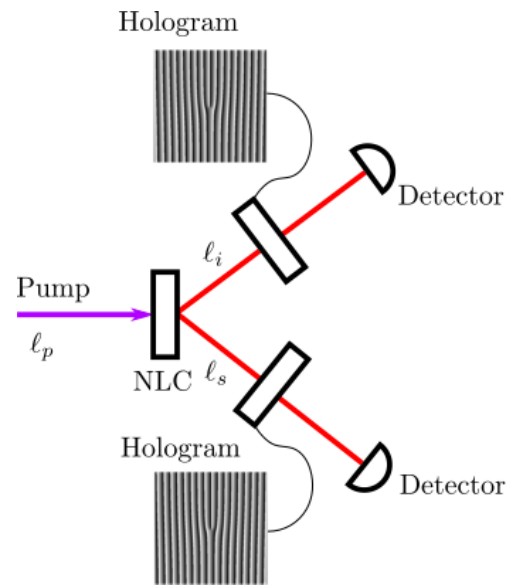

Fig. 17. Schematic representation of OAM generation using a Non-Linear Crystal (NLC). A high intensity pump beam shines an NLC to generate a photon pair. Given that the pump beam is a Gaussian beam, projecting one photon on a particular $\ell$ value, projects its partner on $-\ell$.

In this way, we can express a Fock state in the LG mode as

$$
\left|n_{\ell, p}\right\rangle=\frac{\left(\hat{a}_{k_{0}, \lambda, \ell, p}^{\dagger}\right)^{n}}{\sqrt{n_{\ell, p} !}}|0\rangle .
$$

If we want to study a single photon in the LG mode, then we have

$$
\left|1_{\ell, p}\right\rangle=\hat{a}_{k_{0}, \lambda, \ell, p}^{\dagger}|0\rangle,
$$

where a common notation (assuming a single excitation) is to express the value of $\ell$ and $p$ inside the ket. This means

$$
|\ell, p\rangle=\left|1_{\ell, p}\right\rangle
$$

and considering the latter notation then we have

$$
\left\langle\ell, p \mid \ell^{\prime}, p^{\prime}\right\rangle=\delta_{\ell, \ell^{\prime}} \delta_{p, p^{\prime}} .
$$

\section{Single-photon OAM Generation and Detection Methods}

It is common to generate single photons carrying OAM through the use of a Non-Linear Crystal (NLC) in a process known as Spontaneous Parametric Down Conversion (SPDC) [111]. In this process, a high power laser (and typically with high frequency) shines an NLC and parametrically generates two lower-frequency photons. For convenience, we label those photons as a pump (for the high-frequency input beam), idler, and signal (for the two photons coming out the NLC). This source of single photons is also a source of entanglement. Indeed, the correlations between entangled photons can exist in arrival times, polarization, and momentum. Moreover, one can intuitively expect that since linear momentum is conserved, orbital angular momentum should be conserved too. This led Zeilinger and colleagues to explore this idea, where the photon pairs produced by the non-linear crystal also conserved OAM [118]. For example, if we use a Gaussian beam $\left(\ell_{p}=0\right)$ as the input, then after the NLC each photon carries -say $\ell_{i}$ and $\ell_{s}$, with the condition that $\ell_{p}=\ell_{i}+\ell_{s}$. In this manner, when we project one photon on a particular $\ell$ value, the other photon will possess $-\ell$ (see Fig. 17). This was experimentally verified and since then has been extensively explored for the generation of single photons carrying OAM, entanglement in higher-order modes, as well as non-diffracting and Ince-Gaussian modes [119]-[123]. The issue now is how to project and measure the OAM on a single photon. For that, some ideas are in place. The most 
straightforward way is to think about the typical strategies to generate OAM beams, namely SPP or forked holograms. If we take a Gaussian beam to illuminate either an SPP or a forked hologram, we will surely generate an OAM mode. Then we can think about reversing that process, that is, taking the OAM mode generated by the SPP or the forked hologram and send it back to the same device, as schematically shown in Fig. 8. We can easily see that reversing the process will lead to the same Gaussian input beam. This idea was used to experimentally measure the OAM in a pair of entangled photons [118].

(a)

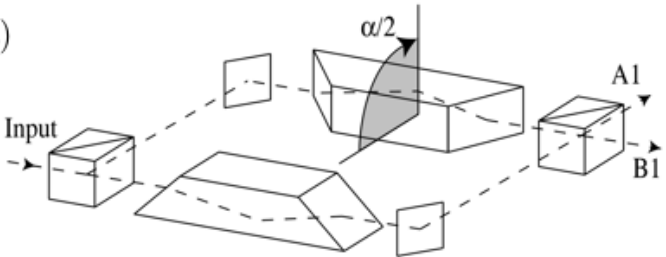

(b)

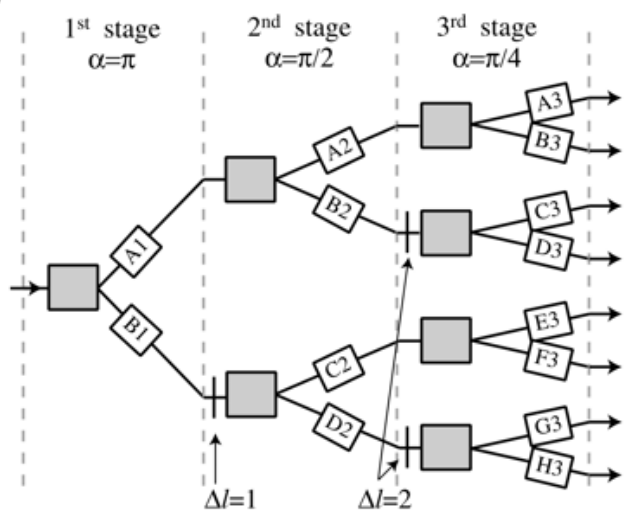

(c)
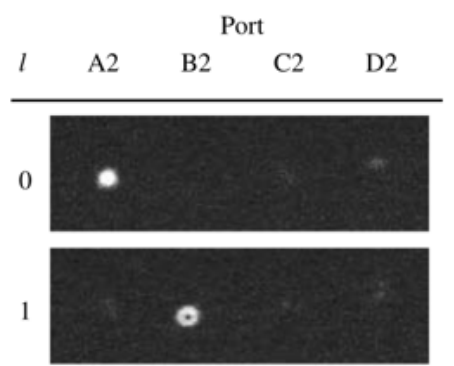

2
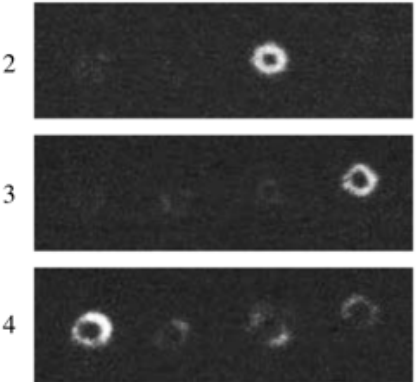

Fig. 18. (a) Modified Mach-Zehnder interferometer with Dove prisms in each arm. By setting an appropriate angle on one of the Dove prisms, we can efficiently sort single photons carrying OAM. (b) Schematic representation to sort higher-order OAM modes. Each gray box, corresponds to on Mach-Zehnder interferometer (as in (a)) (c) Experimental results from [124]. We can see how each photon carrying OAM is sorted to a particular output port. (Adapted from [124])

In terms of measurement, there are some remarkable studies that address this problem in an efficient way. In 2002, Leach and colleagues presented an experimental scheme to sort single photons carrying OAM by using Mach-Zehnder (MZ) interferometers and two Dove prisms, one in each arm, as depicted in Fig. 18(a) [124]. This interferometric technique uses the fact that a Dove prism adds a phase factor dependent on the angle $\alpha$, and particularly for OAM modes, also depends on the $\ell$ value. This means that placing two Dove prisms and properly setting their relative angle will lead to constructive interference in one of the output ports of the MZ interferometer. Cascading many interferometers will allow us to sort single photons by their $\ell$ values (see Fig. 18 (b)-(c)).

Another interesting approach was published in 2010 [125], [126]. In that work, the idea studied a $\log$-polar transformation of the OAM mode, resulting in an unwrapping, i.e. the azimuthal phase variation of the mode is transformed to a linear phase change. To achieve this, two refractive devices are in place (see Fig. 19). The first element unwraps the input mode (left in Fig. 19(a)), whereas the second surface (right in Fig. 19 (a)) corrects the output light. After the second element, a lens is placed to direct the mode to an $\ell$-dependent position in the Fourier plane. The refractive elements have the following phase profiles

$$
\begin{gathered}
\phi_{1}(x, y)=\frac{2 \pi a}{\lambda f}\left[y \tan ^{-1}(y / x)-x \ln \left(\frac{\sqrt{x^{2}+y^{2}}}{b}\right)+x\right], \\
\phi_{2}(u, v)=-\frac{2 \pi a b}{\lambda f} \exp (-u / a) \cos (v / a),
\end{gathered}
$$


where $\lambda$ is the wavelength, $(x, y)$ and $(u, v)$ are the Cartesian coordinates in the input and output planes. $f$ is the focal distance of the lens, $a=d / 2 \pi$ with $d$ related to the length of the transformed beam and $b$ is a parameter that translates the output beam in the $u$ direction. The required phase elements can be observed in Fig. 19.(a), as well as an schematic experimental setup in in Fig. 19.b). Please note that both schemes previously commented are in principle $100 \%$ efficient.

(a)

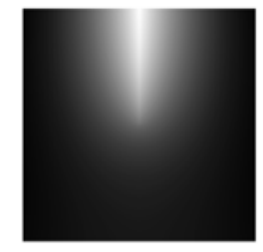

(b)

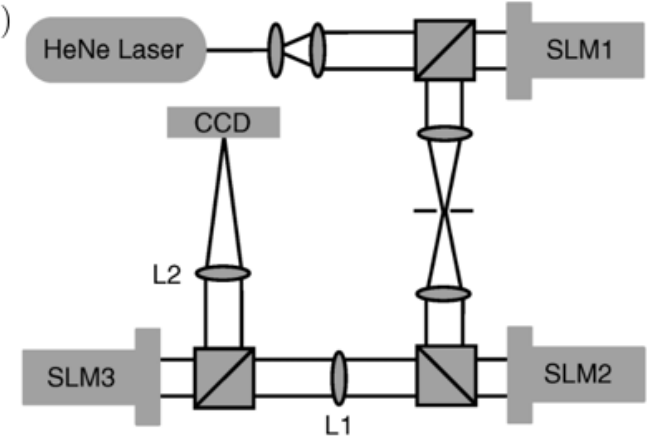

(c)
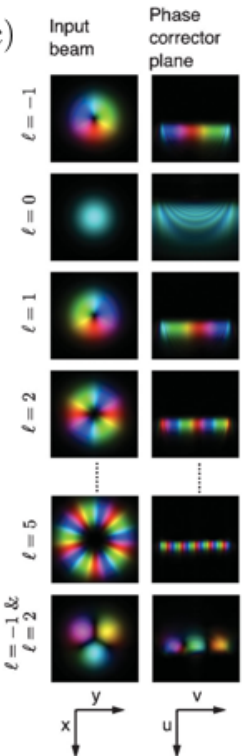

Modeled

detector
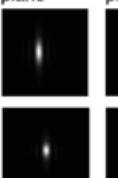

,
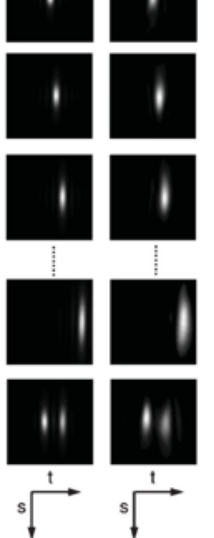

Fig. 19. Efficient detection of OAM states using a Log-Polar transformation. (a) Phase profiles of the refractive elements that constitute the OAM mode sorter. (b) Experimental setup. Notice that both phase elements were programmed on an SLM. (c) Experimental results. Observe how each $\ell$ value is sorted to a particular lateral position at the detector plane. (Adapted from [125])

\section{E. Applications in quantum cryptography}

The higher dimensionality that OAM modes possess is desirable in many situations, namely, sharing or protecting information. This higher dimensionality can be seen as a bigger alphabet available, where each "letter" corresponds to a particular OAM mode. This immediately brings the idea of increasing the capacity to share information or make more robust and secure communications through quantum cryptographic schemes.

The typical players in the context of information sharing are Alice (A), Bob (B), and Eve (E). Alice and Bob want to share information through a channel, while Eve attempts to intercept their messages. Classically speaking, the one-time pad is an encryption technique (cipher) with a message $m \in\{0,1\}^{n}$ and a random key $k \in\{0,1\}^{n}$ such that [127]

$$
\begin{gathered}
E(k, m)=k \oplus m=e, \\
D(k, e)=k \oplus e=m,
\end{gathered}
$$

where $E(\cdot)$ is the encryption, $D(\cdot)$ the decryption, $e$ is the encrypted message and $\oplus$ is the addition modulo 2 or XOR. An interesting feature of this cipher is that it has perfect secrecy given that the length of the key is bigger or equal to the length of the message [128]. This imposes the condition of a long enough key, but also the fact that both parties (Alice and Bob) must possess the key, but not any eavesdropper (Eve). This last point is where quantum key distribution (QKD) comes into play. The idea 


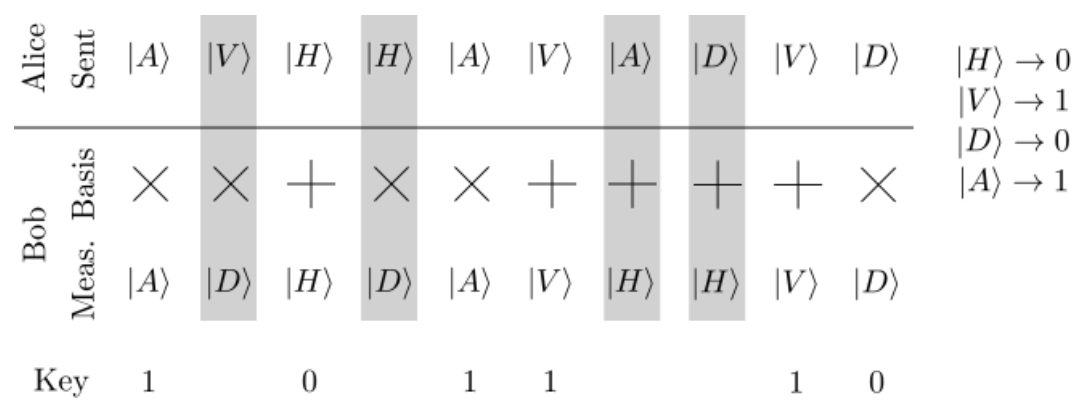

Fig. 20. Schematic representation of the BB84 QKD protocol. The shaded parts represent the discarded bits.

now is to generate a random key and securely distribute it among Alice and Bob, once both have the key, they can secretly share information using, for example, the one-time pad.

We can categorize quantum key distribution protocols according to the property exploited: prepare and measure (for example, BB84 [129]) and entanglement based (for example, E91 [130]) protocols. In both cases, the use of OAM modes presents the advantage of a larger alphabet (in principle unbounded) that allows us to increase the coding density as well as a higher security margin. For simplicity, let us describe the BB84 protocol using a two dimensional Hilbert space (polarization). In this protocol, Alice sends single photons with either horizontal or vertical (HV-basis), diagonal, or anti-diagonal (DA-basis) polarization state through a quantum channel. Then Bob selects a basis at random (HV or DA) and measures the photons sent by Alice. After Bob measures all the photons, he communicates (using a classical channel) the basis chosen by him. Alice then responds back by saying where the basis was correctly picked. Finally, Alice and Bob discard the photons where the basis did not match and obtain a secure key (see Fig. 20). As stated before, we can increase the amount of information by using a larger Hilbert space. This indeed has been done using OAM modes [131]-[133], and vector vortex states [105], [134], increasing thus the efficiency of the protocol. Let us deepen the latter.

In Sec. V, we discussed modes with non-homogeneous polarization distributions across the transverse plane, the so-called vector modes. It turns out that it is possible to generate single photons with such exotic properties [135]. That is, we can experimentally create the single photon state

$$
|\psi\rangle_{\ell, \theta}=\frac{1}{\sqrt{2}}(|\ell\rangle|R\rangle+\exp (i \theta)|-\ell\rangle|L\rangle),
$$

where $\theta \in\{0, \pi\}, R$ stands for right- and $L$ for left-handed polarization. Notice that for a fixed $\ell$ value, we have four orthonormal vector modes, and as such, we also have a mutually unbiased counterpart. Within this context let us define our computational basis for the vector modes

$$
\begin{aligned}
|00\rangle_{v} & =\frac{1}{\sqrt{2}}(|\ell\rangle|R\rangle+|-\ell\rangle|L\rangle), \\
|01\rangle_{v} & =\frac{1}{\sqrt{2}}(|\ell\rangle|R\rangle-|-\ell\rangle|L\rangle), \\
|10\rangle_{v} & =\frac{1}{\sqrt{2}}(|\ell\rangle|L\rangle+|-\ell\rangle|R\rangle), \\
|11\rangle_{v} & =\frac{1}{\sqrt{2}}(|\ell\rangle|L\rangle-|-\ell\rangle|R\rangle),
\end{aligned}
$$

and the corresponding mutually unbiased basis

$$
|00\rangle_{s}=|-\ell\rangle|D\rangle
$$




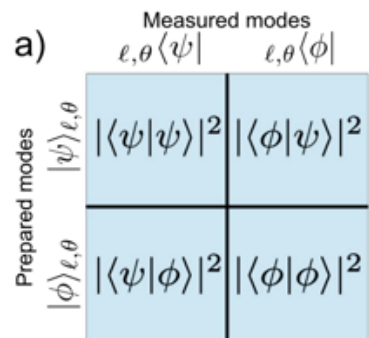

b)

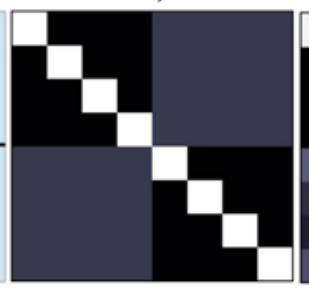

e)

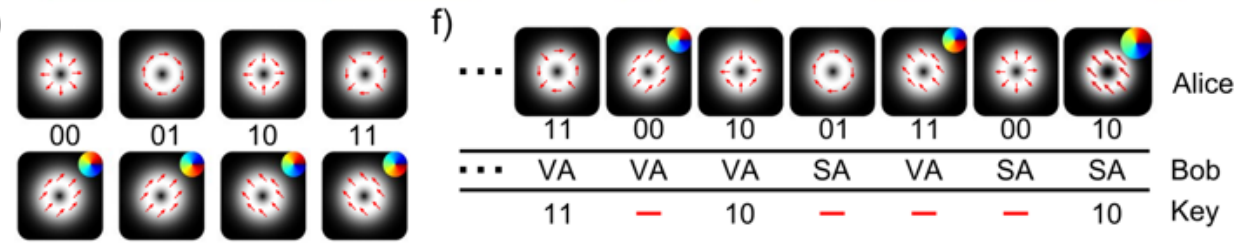

g)

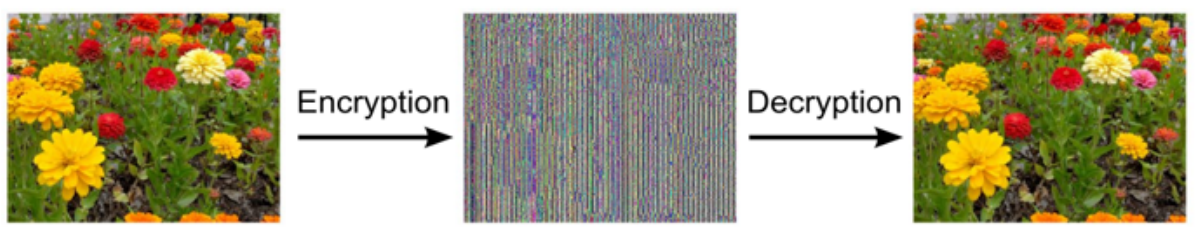

Fig. 21. Schematic representation of the QKD protocol with vector modes. (a)-(d) Schematic and theoretical inner product measurements for the subspace $\ell= \pm 1$ and $\ell= \pm 10$. (e) Vector basis (up) and Scalar basis (down). (f) Alice randomly sends scalar and vector modes, which Bob measures using either a scalar (SA) or vector (VA) detector. In a classical call, both agree which bits remove. (g) Depicts an example of encryption/decryption using a 98 bit long key generated with this approach. (Adapted from [105])

$$
\begin{gathered}
|01\rangle_{s}=|\ell\rangle|D\rangle, \\
|10\rangle_{s}=|-\ell\rangle|A\rangle, \\
|11\rangle_{s}=|\ell\rangle|A\rangle,
\end{gathered}
$$

where $D$ and $A$ represent the diagonal and antidiagonal polarization states, whereas the subscript $v$ and $s$ stand for vector and scalar, respectively. Notice that the vector and scalar basis defined in Eqs. (73) - 80) are analogous to the basis exemplified in Fig. 20. Alice randomly prepares and sends a photon. Bob randomly selects either a scalar or vector detector [105]. Then, Alice and Bob discard the mismatched basis in a classical channel and thus remove the corresponding bits. Finally, both have a randomly generated secret key, which was used to transfer information, as shown in Fig. 21.

\section{SUMMARY}

OAM has seen tremendous progress in various fields since its initial discovery. We started this article by introducing different mode bases with OAM dependence, and we particularly described the main techniques to generate and detect OAM modes derived from the LG mode family. We then focused on different OAM use cases in communication. In a later section, vector OAM carrying beams were discussed. Finally, we discuss the contributions of OAM in quantum optics.

Several other mode bases offer unlimited orthogonal modes that can be harnessed. Still, more research should be done to cope with the different challenges mainly related to the generation and detection of those light modes and understand their propagation dynamics. 


\section{REFERENCES}

[1] L. Allen, M. W. Beijersbergen, R. J. C. Spreeuw, and J. P. Woerdman, "Orbital angular momentum of light and the transformation of Laguerre-Gaussian laser modes," Physical Review A, vol. 45, pp. 8185-8189, 1992.

[2] F. Tamburini, B. Thidé, G. Molina-Terriza, and G. Anzolin, "Twisting of light around rotating black holes," Nature Physics, vol. 7, pp. 195-197, 2011.

[3] M. Padgett and R. Bowman, "Tweezers with a twist," Nature Photonics, vol. 5, p. 343-348, 2011.

[4] B. Jack, J. Leach, J. Romero, S. Franke-Arnold, M. Ritsch-Marte, S. M. Barnett, and M. J. Padgett, "Holographic ghost imaging and the violation of a bell inequality," Physical Review Letters, vol. 103, p. 083602, 2009.

[5] N. Uribe-Patarroyo, A. Fraine, D. S. Simon, O. Minaeva, and A. V. Sergienko, "Object identification using correlated orbital angular momentum states," Physical Review Letters, vol. 110, p. 043601, Jan 2013.

[6] A. Mair, A. Vaziri, G. Weihs, and A. Zeilinger, "Entanglement of the orbital angular momentum states of photons," Nature, vol. 412, p. 313-316, 2001.

[7] A. E. Willner, H. Huang, Y. Yan, Y. Ren, N. Ahmed, G. Xie, C. Bao, L. Li, Y. Cao, Z. Zhao, J. Wang, M. P. J. Lavery, M. Tur, S. Ramachandran, A. F. Molisch, N. Ashrafi, and S. Ashrafi, "Optical communications using orbital angular momentum beams," Advances in Optics and Photonics, vol. 7, no. 1, pp. 66-106, 2015.

[8] A. Trichili, K. Park, M. Zghal, B. S. Ooi, and M. Alouini, "Communicating using spatial mode multiplexing: Potentials, challenges, and perspectives,” IEEE Communications Surveys Tutorials, vol. 21, no. 4, pp. 3175-3203, Fourthquarter 2019.

[9] A. E. Siegman, lasers. University Science Books, 1986.

[10] M. Beijersbergen, L. Allen, H. van der Veen, and J. Woerdman, "Astigmatic laser mode converters and transfer of orbital angular momentum," Optics Communications, vol. 96, no. 1, pp. 123 - 132, 1993.

[11] M. A. Bandres and J. C. Gutiérrez-Vega, "Ince-gaussian beams," Optics Letters, vol. 29, no. 2, pp. 144-146, 2004.

[12] J. Durnin, J. J. Miceli, and J. H. Eberly, "Diffraction-free beams," Physical Review Letters, vol. 58, pp. 1499-1501, 1987.

[13] M. Beijersbergen, R. Coerwinkel, M. Kristensen, and J. Woerdman, "Helical-wavefront laser beams produced with a spiral phaseplate," Optics Communications, vol. 112, no. 5, pp. 321 - 327, 1994.

[14] L. Marrucci, C. Manzo, and D. Paparo, "Optical spin-to-orbital angular momentum conversion in inhomogeneous anisotropic media," Physical Review Letters, vol. 96, p. 163905, 2006.

[15] A. Forbes, A. Dudley, and M. McLaren, "Creation and detection of optical modes with spatial light modulators," Advances in Optics and Photonics, vol. 8, no. 2, pp. 200-227, 2016.

[16] S. Zheng, L. Zha, J. Lu, X. Zeng, Y. Li, S. Xu, and D. Fan, "Liquid crystal-based order electrically controlled q-plate system," Opt. Express, vol. 27, no. 11, pp. 16103-16110, May 2019.

[17] C. Rosales-Guzmán and A. Forbes, How to Shape Light with Spatial Light Modulators. SPIE Press, 2017 , vol. SL30.

[18] M. Mirhosseini, O. S. M. na Loaiza, C. Chen, B. Rodenburg, M. Malik, and R. W. Boyd, "Rapid generation of light beams carrying orbital angular momentum," Optics Express, vol. 21, no. 25, pp. 30 196-30203, Dec 2013.

[19] S. Scholes, R. Kara, J. Pinnell, V. Rodríguez-Fajardo, and A. Forbes, "Structured light with digital micromirror devices: a guide to best practice," Optical Engineering, vol. 59, no. 04, p. 1, nov 2019. [Online]. Available: https://www.spiedigitallibrary.org/journals/optical-engineering/volume-59/issue-04/041202/ Structured-light-with-digital-micromirror-devices--a-guide-to/10.1117/1.OE.59.4.041202.full

[20] M. A. Cox and A. V. Drozdov, "Converting a Texas Instruments DLP4710 DLP evaluation module into a spatial light modulator," Applied Optics, vol. 60, no. 2, pp. 465-469, 2021.

[21] E. Karimi, S. A. Schulz, I. D. Leon, H. Qassim, J. Upham, and R. W. Boyd, "Generating optical orbital angular momentum at visible wavelengths using a plasmonic metasurface," Light and Science Applications, no. e167, 2014.

[22] M. P. J. Lavery, D. J. Robertson, A. Sponselli, J. Courtial, N. K. Steinhoff, G. A. Tyler, A. E. Wilner, and M. J. Padgett, "Efficient measurement of an optical orbital-angular-momentum spectrum comprising more than 50 states," New Journal of Physics, vol. 15, no. 1, p. 013024, jan 2013.

[23] J. Pinnell, I. Nape, B. Sephton, M. Cox, V. Rodriguez-Fajardo, and A. Forbes, "Modal analysis of structured light with spatial lightmodulators: a practical tutorial," Journal of the Optical Society of America A, vol. 37, no. 11, pp. 146-160, 2020.

[24] M. A. Cox, E. Toninelli, L. Cheng, M. J. Padgett, and A. Forbes, "A High-Speed, Wavelength Invariant, Single-Pixel Wavefront Sensor With a Digital Micromirror Device," IEEE Access, vol. 7, pp. 85 860-85 866, 2019.

[25] P. J. Winzer, "Making spatial multiplexing a reality," Nature Photonics, vol. 8, no. 5, pp. 345-348, apr 2014.

[26] M. Krenn, R. Fickler, M. Fink, J. Handsteiner, M. Malik, T. Scheidl, R. Ursin, and A. Zeilinger, "Communication with spatially modulated light through turbulent air across vienna," New Journal of Physics, vol. 16, no. 11, p. $113028,2014$.

[27] M. Krenn, J. Handsteiner, M. Fink, R. Fickler, R. Ursin, M. Malik, and A. Zeilinger, "Twisted light transmission over 143 km," Proceedings of the National Academy of Sciences, vol. 113, no. 48, pp. 13648-13 653, 2016.

[28] A. Ragheb, W. Saif, A. Trichili, I. Ashry, M. A. Esmail, M. Altamimi, A. Almaiman, E. Altubaishi, B. S. Ooi, M.-S. Alouini, and S. Alshebeili, "Identifying structured light modes in a desert environment using machine learning algorithms," Optics Express, no. 7, pp. 9753-9763, 2020.

[29] A. Trichili, C. B. Issaid, B. S. Ooi, and M.-D. Alouini, "A CNN based structured light communication scheme for internet of underwater things applications," IEEE Internet of Things Journal, vol. 7, no. 10, pp. 10 038-10047, 2020.

[30] G. Gibson, J. Courtial, M. J. Padgett, M. Vasnetsov, V. Pas'ko, S. M. Barnett, and S. Franke-Arnold, "Free-space information transfer using light beams carrying orbital angular momentum,” Optics Express, vol. 12, no. 22, pp. 5448-5456, 2004. 
[31] J. Wang, J.-Y. Yang, I. M. Fazal, N. Ahmed, Y. Yan, H. Huang, Y. Ren, Y. Yue, S. Dolinar, M. Tur, and A. E. Willner, "Terabit free-space data transmission employing orbital angular momentum multiplexing," Nature Photonics, vol. 6, no. 7, pp. 488-496, 2012.

[32] H. Huang, G. Xie, Y. Yan, N. Ahmed, Y. Ren, Y. Yue, D. Rogawski, M. J. Willner, B. I. Erkmen, K. M. Birnbaum, S. J. Dolinar, M. P. J. Lavery, M. J. Padgett, M. Tur, and A. E. Willner, "100 Tbit/s free-space data link enabled by three-dimensional multiplexing of orbital angular momentum, polarization, and wavelength," Optics Letters, vol. 39, no. 2, pp. 197-200, 2014.

[33] J. Wang, S. Li, M. Luo, J. Liu, L. Zhu, C. Li, D. Xie, Q. Yang, S. Yu, J. Sun, X. Zhang, W. Shieh, and A. E. Willner, "N-dimentional multiplexing link with 1.036-Pbit/s transmission capacity and 112.6-bit/s/Hz spectral efficiency using OFDM-8QAM signals over 368 WDM pol-muxed 26 OAM modes," in European Conference on Optical Communication, ECOC. Institute of Electrical and Electronics Engineers Inc., 2014.

[34] Y. Ren, Z. Wang, P. Liao, L. Li, G. Xie, H. Huang, Z. Zhao, Y. Yan, N. Ahmed, A. Willner, M. P. J. Lavery, N. Ashrafi, S. Ashrafi, R. Bock, M. Tur, I. B. Djordjevic, M. A. Neifeld, and A. E. Willner, "Experimental characterization of a $400 \mathrm{Gbit} / \mathrm{s}$ orbital angular momentum multiplexed free-space optical link over $120 \mathrm{~m}$," Optics Letters, vol. 41, no. 3, pp. 622-625, 2016.

[35] L. Li, R. Zhang, Z. Zhao, G. Xie, P. Liao, K. Pang, H. Song, C. Liu, Y. Ren, G. Labroille, P. Jian, D. Starodubov, B. Lynn, R. Bock, M. Tur, and A. E. Willner, "High-capacity free-space optical communications between a ground transmitter and a ground receiver via a UAV using multiplexing of multiple orbital-angular-momentum beams," Scientific Reports, vol. 7, no. $17427,2017$.

[36] D. J. Richardson, J. M. Fini, and L. E. Nelson, "Space-division multiplexing in optical fibres," Nature Photonics, vol. 7, p. 354-362, 2013.

[37] S. Berdagué and P. Facq, "Mode division multiplexing in optical fibers," Appl. Opt., vol. 21, no. 11, pp. 1950-1955, 1982.

[38] D. Marcuse, Theory of Dielectric Optical Waveguides, 2nd ed. Academic Press, 1991.

[39] N. Bozinovic, Y. Yue, Y. Ren, M. Tur, P. Kristensen, H. Huang, A. E. Willner, and S. Ramachandran, "Terabit-scale orbital angular momentum mode division multiplexing in fibers," Science, vol. 340, no. 6140, pp. 1545-1548, 2013.

[40] L. A. Rusch, M. Rad, K. Allahverdyan, I. Fazal, and E. Bernier, "Carrying data on the orbital angular momentum of light," IEEE Communications Magazine, vol. 56, no. 2, pp. 219-224, 2018.

[41] A. Wang, L. Zhu, L. Wang, J. Ai, S. Chen, and J. Wang, "Directly using 8.8-km conventional multi-mode fiber for 6-mode orbital angular momentum multiplexing transmission,” Optics Express, vol. 26, no. 8, pp. 10038-10047, 2018.

[42] N. Saeed, A. Celik, T. Y. Al-Naffouri, and M.-S. Alouini, "Underwater optical wireless communications, networking, and localization: A survey," Ad Hoc Networks, vol. 94, p. 101935, 2019.

[43] J. Baghdady, K. Miller, K. Morgan, M. Byrd, S. Osler, R. Ragusa, W. Li, B. M. Cochenour, and E. G. Johnson, "Multigigabit/s underwater optical communication link using orbital angular momentum multiplexing," Optics Express, vol. 24, no. 9, pp. 9794-9805, 2016.

[44] Y. Ren, L. Li, Z. Wang, S. M. Kamali, E. Arbabi, A. Arbabi, Z. Zhao, G. Xie, Y. Cao, N. Ahmed, Y. Yan, C. Liu, A. J. Willner, S. Ashrafi, M. Tur, A. Faraon, and A. E. Willner, "Orbital angular momentum-based space division multiplexing for high-capacity underwater optical communications," Scientific Reports, vol. 6, no. 33306, 2016.

[45] Y. Chen, W.-G. Shen, Z.-M. Li, C.-Q. Hu, Z.-Q. Yan, Z.-Q. Jiao, J. Gao, M.-M. Cao, K. Sun, and X.-M. Jin, "Underwater transmission of high-dimensional twisted photons over 55 meters," PhotoniX, vol. 1, no. 5, 2020.

[46] B. Thidé, H. Then, J. Sjöholm, K. Palmer, J. Bergman, T. D. Carozzi, Y. N. Istomin, N. H. Ibragimov, and R. Khamitova, "Utilization of photon orbital angular momentum in the low-frequency radio domain," Physical Review Letters, vol. 99, p. 087701, 2007.

[47] F. Tamburini, E. Mari, A. Sponselli, B. Thidé, A. Bianchini, and F. Romanato, "Encoding many channels on the same frequency through radio vorticity: first experimental test," New Journal of Physics, vol. 14, no. 3, p. 033001, 2012.

[48] Y. Yana, G. Xie, M. P. J. Lavery, H. Huang, N. Ahmed, C. Bao, Y. Ren, Y. Cao, L. Li, Z. Zhao, A. F. Molisch, M. Tur, M. J. Padgett, and A. E. Willner, "High-capacity millimetre-wave communications with orbital angular momentum multiplexing," Nature Communications, vol. 5, 2012.

[49] S. M. Mohammadi, L. K. S. Daldorff, J. E. S. Bergman, R. L. Karlsson, B. Thide, K. Forozesh, T. D. Carozzi, and B. Isham, "Orbital angular momentum in radio-a system study," IEEE Transactions on Antennas and Propagation, vol. 58, no. 2, pp. 565-572, 2010.

[50] H. Wu, Y. Yuan, Z. Zhang, and J. Cang, "Uca-based orbital angular momentum radio beam generation and reception under different array configurations," in 2014 Sixth International Conference on Wireless Communications and Signal Processing (WCSP), 2014, pp. 1-6.

[51] M. J. Padgett, F. M. Miatto, M. P. J. Lavery, A. Zeilinger, and R. W. Boyd, "Divergence of an orbital-angular-momentumcarrying beam upon propagation," New Journal of Physics, vol. 17, no. 2, p. 023011, 2015.

[52] M. A. Cox, N. Mphuthi, I. Nape, N. Mashaba, L. Cheng, and A. Forbes, "Structured Light in Turbulence," IEEE Journal of Selected Topics in Quantum Electronics, vol. 27, no. 2, pp. 1-21, mar 2021. [Online]. Available: https://ieeexplore.ieee.org/document/9198098/

[53] J. A. Anguita, M. A. Neifeld, and B. V. Vasic, "Turbulence-induced channel crosstalk in an orbital angular momentummultiplexed free-space optical link," Appl. Opt., vol. 47, no. 13, pp. 2414-2429, 2008. 
[54] A. Trichili, M. A. Cox, B. S. Ooi, and M.-S. Alouini, "Roadmap to free space optics," Journal of the Optical Society of America B, vol. 37, no. 11, pp. A184-A201, 2020.

[55] Y. Yan, L. Li, G. Xie, C. Bao, P. Liao, H. Huang, Y. Ren, N. Ahmed, Z. Zhao, Z. Wang, N. Ashrafi, S. Ashrafi, S. Talwar, S. Sajuyigbe, M. Tur, A. F. Molisch, and A. E. Willner, "Multipath effects in millimetre-wave wireless communication using orbital angular momentum multiplexing," Scientific Reports, 2016.

[56] Y. Ren, G. Xie, H. Huang, N. Ahmed, Y. Yan, L. Li, C. Bao, M. P. J. Lavery, M. Tur, M. A. Neifeld, R. W. Boyd, J. H. Shapiro, and A. E. Willner, "Adaptive-optics-based simultaneous pre- and post-turbulence compensation of multiple orbital-angular-momentum beams in a bidirectional free-space optical link," Optica, vol. 1, no. 6, pp. 376-382, Dec 2014.

[57] H. Song, H. Song, R. Zhang, K. Manukyan, L. Li, Z. Zhao, K. Pang, C. Liu, A. Almaiman, R. Bock, B. Lynn, M. Tur, and A. E. Willner, "Experimental mitigation of atmospheric turbulence effect using pre-signal combining for uni- and bi-directional free-space optical links with two 100-Gbit/s OAM-multiplexed channels," Journal of Lightwave Technology, vol. 38, no. 1, pp. 82-89, 2020.

[58] E. M. Amhoud, A. Trichili, B. S. Ooi, and M. Alouini, "Oam mode selection and space-time coding for atmospheric turbulence mitigation in fso communication," IEEE Access, vol. 7, pp. 88 049-88 057, 2019.

[59] M. A. Cox, L. Maqondo, R. Kara, G. Milione, L. Cheng, and A. Forbes, "The Resilience of Hermite- and Laguerre-Gaussian modes in turbulence," Journal of Lightwave Technology, vol. 37, no. 16, pp. 3911-3917, 2019.

[60] M. A. Cox, C. Rosales-Guzmán, M. P. J. Lavery, D. J. Versfeld, and A. Forbes, "On the resilience of scalar and vector vortex modes in turbulence," Optics Express, vol. 24, no. 16, p. 18105, 2016.

[61] M. A. Cox, L. Cheng, C. Rosales-Guzmán, and A. Forbes, "Modal Diversity for Robust Free-Space Optical Communications," Physical Review Applied, vol. 10, no. 2, p. 024020, 2018.

[62] X. Gu, L. Chen, and M. Krenn, "Phenomenology of complex structured light in turbulent air," Optics Express, vol. 28, no. 8, p. $11033,2020$.

[63] G. R. Mehrpoor, M. Safari, and B. Schmauss, "Free space optical communication with spatial diversity based on orbital angular momentum of light," in 2015 4th International Workshop on Optical Wireless Communications (IWOW). IEEE, 2015, pp. 78-82.

[64] S. Huang, G. R. Mehrpoor, and M. Safari, "Spatial-Mode Diversity and Multiplexing for FSO Communication With Direct Detection," IEEE Transactions on Communications, vol. 66, no. 5, pp. 2079-2092, 2018.

[65] C. Zhang and L. Ma, "Millimetre wave with rotational orbital angular momentum," Scientific Reports, vol. 6, no. 31921, 2016.

[66] Y. Yan, L. Li, G. Xie, M. Ziyadi, A. M. Ariaei, Y. Ren, O. Renaudin, Z. Zhao, Z. Wang, C. Liu, S. Sajuyigbe, S. Talwar, S. Ashrafi, A. F. Molisch, and A. E. Willner, "OFDM over mm-wave OAM channels in a multipath environment with intersymbol interference," in 2016 IEEE Global Communications Conference (GLOBECOM), 2016, pp. 1-6.

[67] A. Trichili, C. Rosales-Guzmán, A. Dudley, B. Ndagano, M. Z. Amine Ben Salem, and A. Forbes, "Optical communication beyond orbital angular momentum," Scienctific Rports, vol. 6, no. 27674, 2016.

[68] G. Xie, Y. Ren, Y. Yan, H. Huang, N. Ahmed, L. Li, Z. Zhao, C. Bao, M. Tur, S. Ashrafi, and A. E. Willner, "Experimental demonstration of a 200 -gbit/s free-space optical link by multiplexing laguerre-gaussian beams with different radial indices," Optics Letters, vol. 41, no. 15, pp. 3447-3450, 2016.

[69] B. Ndagano, N. Mphuthi, G. Milione, and A. Forbes, "Comparing mode-crosstalk and mode-dependent loss of laterally displaced orbital angular momentum and Hermite-Gaussian modes for free-space optical communication," Optics Letters, vol. 42, no. 20, p. 4175, 2017.

[70] L. Li, H. Song, R. Zhang, Z. Zhao, C. Liu, K. Pang, H. Song, J. Du, A. N. Willner, A. Almaiman, B. Lynn, R. Bock, M. Tur, and A. E. Willner, "Demonstration of Both Mode and Space Diversity in a 100-Gbit/s QPSK Free-Space Optical Link to Increase System Tolerance to Turbulence," in Optical Fiber Communication Conference (OFC) 2019. Washington, D.C.: OSA, 2019, p. W4A.5.

[71] H. Li, D. B. Phillips, X. Wang, Y.-L. D. Ho, L. Chen, X. Zhou, J. Zhu, S. Yu, and X. Cai, "Orbital angular momentum vertical-cavity surface-emitting lasers," Optica, vol. 2, no. 6, pp. 547-552, 2015.

[72] Z. Zhang, X. Qiao, B. Midya, K. Liu, J. Sun, T. Wu, W. Liu, R. Agarwal, J. M. Jornet, S. Longhi, N. M. Litchinitser, and L. Feng, "Tunable topological charge vortex microlaser," Science, vol. 368, no. 6492, pp. 760-763, 2020.

[73] E. Stegenburgs, A. Bertoncini, A. Trichili, M. S. Alias, T. K. Ng, M.-S. Alouini, C. Liberale, and B. S. Ooi, "Near-infrared OAM communication using 3D-printed microscale spiral phase plates," IEEE Communications Magazine, vol. 57, no. 8, pp. 65-69, 2019.

[74] S. Lightman, G. Hurvitz, R. Gvishi, and A. Arie, "Miniature wide-spectrum mode sorter for vortex beams produced by 3D laser printing," Optica, vol. 4, no. 6, pp. 605-610, 2017.

[75] G. Ruffato, M. Girardi, M. Massari, E. Mafakheri, B. Sephton, P. Capaldo, A. Forbes, and F. Romanato, "A compact diffractive sorter for high-resolution demultiplexing of orbital angular momentum beams," Scientific Reports, vol. 8, no. 10248, 2018.

[76] Y. Mushiake, K. Matsumura, and N. Nakajima, "Generation of radially polarized optical beam mode by laser oscillation," Proceedings of the IEEE, vol. 60, no. 9, pp. 1107-1109, 1972.

[77] D. G. Hall, "Vector-beam solutions of Maxwell's wave equation," Optics Letters, vol. 21, no. 1, p. 9, 1996.

[78] Q. Zhan, "Cylindrical vector beams: from mathematical concepts to applications," Advances in Optics and Photonics, vol. 1, no. 1, pp. 1-57, 2009. 
[79] B. Ndagano, I. Nape, M. A. Cox, C. Rosales-Guzman, and A. Forbes, "Creation and detection of vector vortex modes for classical and quantum communication,” Journal of Lightwave Technology, vol. 36, pp. 292-301, 2017.

[80] G. G. Stokes, "On the composition and resolution of streams of polarized light from different sources," Transactions of the Cambridge Philosophical Society, vol. 9, p. 399, 1851.

[81] G. Milione, H. I. Sztul, D. A. Nolan, and R. R. Alfano, "Higher-order Poincaré sphere, stokes parameters, and the angular momentum of light." Physical Review Letters, vol. 107, no. 5, p. 053601, 2011.

[82] A. Forbes, Laser Beam Propagation: Generation and Propagation of Customized Light, A. Forbes, Ed. CRC Press, 2014.

[83] J. Sakurai and J. Napolitano, Modern Quantum Mechanics. Cambridge University Press, 2017.

[84] Y. Gu, O. Korotkova, and G. Gbur, "Scintillation of nonuniformly polarized beams in atmospheric turbulence," Optics Letters, vol. 34, no. 15, pp. 2261-2263, 2009.

[85] Y. Gu and G. Gbur, "Reduction of turbulence-induced scintillation by nonuniformly polarized beam arrays," Optics Letters, vol. 37, no. 9, pp. 1553-1555, 2012.

[86] W. Cheng, J. W. Haus, and Q. Zhan, "Propagation of vector vortex beams through a turbulent atmosphere," Optics Express, vol. 17, no. 20, pp. 17829-17 836, 2009.

[87] B. Ndagano, R. Brüning, M. McLaren, M. Duparré, and A. Forbes, "Fiber propagation of vector modes," Optics Express, vol. 23, no. 13, pp. 17330-17336, 2015.

[88] D. G. Grier, “A revolution in optical manipulation,” Nature, vol. 424, no. 6950, pp. 810-816, 2003.

[89] Q. Zhan, "Trapping metallic rayleigh particles with radial polarization,” Optics Express, vol. 12, no. 15, pp. 3377-3382, 2004.

[90] N. Bhebhe, P. A. Williams, C. Rosales-Guzmán, V. Rodriguez-Fajardo, and A. Forbes, "A vector holographic optical trap," Scientific Reports, vol. 8, no. 1, pp. 1-9, 2018.

[91] M. Kraus, M. A. Ahmed, A. Michalowski, A. Voss, R. Weber, and T. Graf, "Microdrilling in steel using ultrashort pulsed laser beams with radial and azimuthal polarization," Optics Express, vol. 18, no. 21, pp. 22 305-22 313, 2010.

[92] J. Hamazaki, R. Morita, K. Chujo, Y. Kobayashi, S. Tanda, and T. Omatsu, "Optical-vortex laser ablation,” Optics Express, vol. 18 , no. 3, pp. 2144-2151, 2010

[93] R. Weber, A. Michalowski, M. Abdou-Ahmed, V. Onuseit, V. Rominger, M. Kraus, and T. Graf, "Effects of radial and tangential polarization in laser material processing," Physics Procedia, vol. 12, pp. 21-30, 2011.

[94] K. Toyoda, F. Takahashi, S. Takizawa, Y. Tokizane, K. Miyamoto, R. Morita, and T. Omatsu, "Transfer of light helicity to nanostructures," Physical Review Letters, vol. 110, no. 14, p. 143603, 2013.

[95] L. J. Wong and F. X. Kärtner, "Direct acceleration of an electron in infinite vacuum by a pulsed radially-polarized laser beam," Optics Express, vol. 18, no. 24, pp. 25 035-25 051, 2010.

[96] X. Hao, C. Kuang, T. Wang, and X. Liu, "Effects of polarization on the de-excitation dark focal spot in sted microscopy," Journal of Optics, vol. 12, no. 11, p. 115707, 2010.

[97] R. Chen, K. Agarwal, C. J. Sheppard, and X. Chen, "Imaging using cylindrical vector beams in a high-numerical-aperture microscopy system," Optics Letters, vol. 38, no. 16, pp. 3111-3114, 2013.

[98] P. H. Jones, M. Rashid, M. Makita, and O. M. Maragò, "Sagnac interferometer method for synthesis of fractional polarization vortices." Optics Letters, vol. 34, no. 17, pp. 2560-2, 2009.

[99] A. Forbes, A. Dudley, and M. McLaren, "Creation and detection of optical modes with spatial light modulators," Advances in Optics and Photonics, vol. 8, no. 2, p. 200, 2016.

[100] K. Singh, N. Tabebordbar, A. Forbes, and A. Dudley, "Digital Stokes polarimetry and its application to structured light: tutorial," Journal of the Optical Society of America A, vol. 37, no. 11, p. C33, 2020.

[101] K. J. Mitchell, S. Turtaev, M. J. Padgett, T. Čižmár, and D. B. Phillips, "High-speed spatial control of the intensity, phase and polarisation of vector beams using a digital micro-mirror device," Optics Express, vol. 24, no. 25, p. 29269 , 2016.

[102] C. Rosales-Guzmán, X. B. Hu, A. Selyem, P. Moreno-Acosta, S. Franke-Arnold, R. Ramos-Garcia, and A. Forbes, "Polarisation-insensitive generation of complex vector modes from a digital micromirror device," Scientific Reports, vol. 10, no. 1, pp. 1-9, 2020.

[103] B. Perez-Garcia, C. López-Mariscal, R. I. Hernandez-Aranda, and J. C. Gutiérrez-Vega, "On-demand tailored vector beams," Applied Optics, vol. 56, no. 24, pp. 6967-6972, 2017.

[104] G. Milione, T. A. Nguyen, J. Leach, D. A. Nolan, and R. R. Alfano, "Using the nonseparability of vector beams to encode information for optical communication," Optics letters, vol. 40, no. 21, pp. 4887-4890, 2015.

[105] B. Ndagano, I. Nape, B. Perez-Garcia, S. Scholes, R. I. Hernandez-Aranda, T. Konrad, M. P. Lavery, and A. Forbes, "A deterministic detector for vector vortex states," Scientific Reports, vol. 7, no. 1, pp. 1-8, 2017.

[106] S. Zheng, Y. Li, Q. Lin, X. Zeng, G. Zheng, Y. Cai, Z. Chen, S. Xu, and D. Fan, "Experimental realization to efficiently sort vector beams by polarization topological charge via pancharatnam-berry phase modulation," Photon. Res., vol. 6, no. 5, pp. 385-389, May 2018.

[107] S. Zheng, X. Zeng, H. Shangguan, Y. Cai, X. Pan, S. Xu, X. Yuan, and D. Fan, "Improve polarization topological order sorting with the diffractive splitting method," Optics Letters, vol. 44, no. 4, pp. 795-798, 2019.

[108] D. Clarke and J. Grainger, "Polarized light and optical measurement," American Journal of Physics, vol. 40, pp. 1055-1056, 1971.

[109] B. Ndagano, H. Sroor, M. McLaren, C. Rosales-Guzmán, and A. Forbes, "Beam quality measure for vector beams," Optics Letters, vol. 41, no. 15, p. 3407, 2016. 
[110] M. McLaren, T. Konrad, and A. Forbes, "Measuring the nonseparability of vector vortex beams," Physical Review A Atomic, Molecular, and Optical Physics, vol. 92, no. 2, pp. 1-7, 2015.

[111] L. Mandel, E. Wolf, and C. U. Press, Optical Coherence and Quantum Optics, ser. EBL-Schweitzer. Cambridge University Press, 1995.

[112] S. Barnett and P. Radmore, Methods in Theoretical Quantum Optics, ser. Oxford Series in Optical and Imaging Sciences. Clarendon Press, 2002.

[113] Y. Yamamoto, Quantum Optics. American Cancer Society, 2003.

[114] C. Gerry, P. Knight, and P. Knight, Introductory Quantum Optics. Cambridge University Press, 2005.

[115] G. Grynberg, A. Aspect, C. Fabre, and C. Cohen-Tannoudji, Introduction to Quantum Optics: From the Semi-classical Approach to Quantized Light. Cambridge University Press, 2010.

[116] P. Kok and B. Lovett, Introduction to Optical Quantum Information Processing. Cambridge University Press, 2010.

[117] V. Fock, "Konfigurationsraum und zweite quantelung," Zeitschrift für Physik, vol. 75, no. 9-10, pp. 622-647, 1932.

[118] A. Mair, A. Vaziri, G. Weihs, and A. Zeilinger, "Entanglement of the orbital angular momentum states of photons," Nature, vol. 412, no. 6844, pp. 313-316, 2001.

[119] H. H. Arnaut and G. A. Barbosa, "Orbital and intrinsic angular momentum of single photons and entangled pairs of photons generated by parametric down-conversion," Physical Review Letters, vol. 85, pp. 286-289, Jul 2000.

[120] S. Franke-Arnold, S. M. Barnett, M. J. Padgett, and L. Allen, "Two-photon entanglement of orbital angular momentum states," Physical Review A, vol. 65, p. 033823, Feb 2002.

[121] R. Fickler, G. Campbell, B. Buchler, P. K. Lam, and A. Zeilinger, "Quantum entanglement of angular momentum states with quantum numbers up to 10,010," Proceedings of the National Academy of Sciences, 2016.

[122] M. McLaren, M. Agnew, J. Leach, F. S. Roux, M. J. Padgett, R. W. Boyd, and A. Forbes, "Entangled bessel-gaussian beams," Optics Express, vol. 20, no. 21, pp. 23 589-23 597, Oct 2012.

[123] M. Krenn, R. Fickler, M. Huber, R. Lapkiewicz, W. Plick, S. Ramelow, and A. Zeilinger, "Entangled singularity patterns of photons in ince-gauss modes," Physical Review A, vol. 87, p. 012326, Jan 2013.

[124] J. Leach, M. J. Padgett, S. M. Barnett, S. Franke-Arnold, and J. Courtial, "Measuring the orbital angular momentum of a single photon," Physical Review Letters, vol. 88, p. 257901, Jun 2002.

[125] G. C. G. Berkhout, M. P. J. Lavery, J. Courtial, M. W. Beijersbergen, and M. J. Padgett, "Efficient sorting of orbital angular momentum states of light," Physical Review Letters, vol. 105, p. 153601, Oct 2010.

[126] M. P. J. Lavery, D. J. Robertson, G. C. G. Berkhout, G. D. Love, M. J. Padgett, and J. Courtial, "Refractive elements for the measurement of the orbital angular momentum of a single photon," Optics Express, vol. 20, no. 3, pp. 2110-2115, Jan 2012.

[127] J. Katz and Y. Lindell, Introduction to Modern Cryptography, Second Edition, ser. Chapman \& Hall/CRC Cryptography and Network Security Series. Taylor \& Francis, 2014.

[128] C. E. Shannon, "Communication theory of secrecy systems," The Bell System Technical Journal, vol. 28, no. 4, pp. 656-715, 1949.

[129] C. H. Bennett and G. Brassard, "Quantum cryptography: Public key distribution and coin tossing," Theoretical Computer Science, vol. 560, pp. 7 - 11, 2014, theoretical Aspects of Quantum Cryptography - celebrating 30 years of BB84.

[130] A. K. Ekert, "Quantum cryptography based on bell's theorem," Physical Review Letters, vol. 67, pp. 661-663, Aug 1991.

[131] S. Gröblacher, T. Jennewein, A. Vaziri, G. Weihs, and A. Zeilinger, "Experimental quantum cryptography with qutrits," New Journal of Physics, vol. 8, no. 5, pp. 75-75, may 2006.

[132] M. Mafu, A. Dudley, S. Goyal, D. Giovannini, M. McLaren, M. J. Padgett, T. Konrad, F. Petruccione, N. Lütkenhaus, and A. Forbes, "Higher-dimensional orbital-angular-momentum-based quantum key distribution with mutually unbiased bases," Physical Review A, vol. 88, p. 032305, Sep 2013.

[133] E. Otte, I. Nape, C. Rosales-Guzmán, C. Denz, A. Forbes, and B. Ndagano, "High-dimensional cryptography with spatial modes of light: tutorial," Journal of the Optical Society of America B, vol. 37, no. 11, pp. A309-A323, Nov 2020.

[134] A. Sit, F. Bouchard, R. Fickler, J. Gagnon-Bischoff, H. Larocque, K. Heshami, D. Elser, C. Peuntinger, K. Günthner, B. Heim, C. Marquardt, G. Leuchs, R. W. Boyd, and E. Karimi, "High-dimensional intracity quantum cryptography with structured photons," Optica, vol. 4, no. 9, pp. 1006-1010, Sep 2017.

[135] R. Fickler, R. Lapkiewicz, S. Ramelow, and A. Zeilinger, "Quantum entanglement of complex photon polarization patterns in vector beams," Physical Review A, vol. 89, p. 060301, Jun 2014. 